\title{
ResearchOnline@JCU
}

This is the author-created version of the following work:

Nematollahi, Neda, Weinberg, Justine Lew, Flattery, Jennifer, Goodman, Nigel, Kolev, Spas D., and Steinemann, Anne (2021) Volatile chemical emissions from essential oils with therapeutic claims. Air Quality, Atmosphere and Health, 14 pp. 365-369.

Access to this file is available from:

https://researchonline.jcu.edu.au/64707/

(C) Springer Nature B.V. 2020

Please refer to the original source for the final version of this work: 
Neda Nematollahi, Justine Lew Weinberg, Jennifer Flattery, Nigel Goodman, Spas D. Kolev, Anne Steinemann. 2020. Volatile Chemical Emissions from Essential Oils with Therapeutic Claims. in Air Quality, Atmosphere, and Health. https://doi.org/10.1007/s11869-020-00941-4

\section{Volatile Chemical Emissions from Essential Oils with Therapeutic Claims}

Neda Nematollahi*, Justine Lew Weinberg, Jennifer Flattery, Nigel Goodman, Spas D. Kolev, Anne Steinemann

*Corresponding author: Neda Nematollahi

neda.nematollahi@unimelb.edu.au

\section{Neda Nematollahi*}

Postdoctoral Research Fellow, Department of Infrastructure Engineering, Melbourne School of Engineering, The University of Melbourne, Parkville Victoria 3010 Australia

\section{Justine Lew Weinberg}

MSEHS, CIH, Public Health Institute, contractor to Occupational Health Branch, California Department of Public Health, Richmond California 94804 United States

\section{Jennifer Flattery}

M.P.H., Research Scientist Supervisor, Occupational Health Branch, California Department of Public Health, Richmond California 94804 United States

\section{Nigel Goodman}

Vice Chancellor's Postdoctoral Research Fellow, School of Property Construction and Project Management, RMIT University, Melbourne VIC 3000 Australia

\section{Spas D. Kolev}

Professor of Chemistry, School of Chemistry, The University of Melbourne, Parkville Victoria 3010 Australia

\section{Anne Steinemann}

Professor of Civil Engineering, Department of Infrastructure Engineering, Melbourne School of Engineering, The University of Melbourne, Parkville Victoria 3010 Australia

Professor of Engineering, Chair of Sustainable Infrastructure, College of Science and Engineering, James Cook University, Townsville Queensland 4814 Australia

This is a pre-print of an article published in Air Quality, Atmosphere, and Health. The final authenticated version is available online at: https://doi.org/10.1007/s11869-020-00941-4 


\title{
Volatile Chemical Emissions from Essential Oils with Therapeutic Claims
}

\begin{abstract}
Essential oils emit many volatile organic compounds (VOCs), with some considered potentially hazardous. However, little is known about specific emissions from essential oils that make therapeutic claims for health and well-being. This study investigated VOCs emitted from 14 commercially available essential oils with therapeutic claims, such as beneficial for coughs, colds, flus, relaxation, sleep, tension, headaches, stress, or skin irritation. The essential oils were selected from different brands and types, such as tea tree oil, lavender oil, eucalyptus oil, geranium oil, peppermint oil, bergamot oil, orange oil, and oil blends. Analyses were performed using headspace gas chromatography/mass spectrometry (GC/MS). The analyses found 1,034 VOCs emitted from the 14 essential oils, representing 378 VOC identities. The most prevalent VOCs (in more than $90 \%$ of the oils) were acetaldehyde, alphaphellandrene, alpha-pinene, camphene, limonene, methanol, terpinolene, 3-carene, acetone, beta-phellandrene, ethanol, and gamma-terpinene. Among the 1,034 VOCs emitted, 251 VOCs, representing 60 VOCs identities, are classified as potentially hazardous. The most prevalent potentially hazardous VOCs were acetaldehyde, limonene, methanol, acetone, ethanol, and 3-carene. Toluene was found in more than $70 \%$ of the essential oils. Each of the essential oils emitted 9 or more potentially hazardous VOCs. Fewer than 1\% of all VOCs identified and fewer than $1 \%$ of all potentially hazardous VOCs were listed on any essential oil label, safety data sheet, or website. Results from this study provide new findings on VOC emissions from essential oils with therapeutic claims, which can help to improve public awareness about potential exposures and risks.
\end{abstract}

Keywords: essential oils, therapeutic, emissions, volatile organic compounds, hazardous compounds, fragrance, ingredients 


\section{Introduction}

Essential oils with therapeutic claims are widely used in many public and private places for different purposes, such as aromatherapy, antibacterial effects, and alleviation of cold and flu symptoms. Essential oils, such as tea tree oil, lavender oil, eucalyptus oil, peppermint oil, and orange oil, are complex mixtures of many different compounds (e.g., Milhem et al. 2020; Nematollahi et al. 2018a; Huang et al. 2012).

Numerous studies have examined potential health benefits of essential oils. For example, Shaaban et al. (2012) reviewed bioactivities associated with essential oils, including antibacterial, antiviral, anti-inflammatory, and additional properties. More specifically, Cavanagh and Wilkinson (2002) reviewed the physiological and psychological effects associated with lavender oil.

However, relatively few studies have examined potential hazards of essential oils. Exposure to essential oils has been associated with adverse health effects such as skin irritation and allergic contact dermatitis (Sarkic and Stappen 2018). Milhem et al. (2020) detailed negative effects of essential oils on indoor air quality.

Further, relatively few studies have analyzed VOCs emitted from commercially available essential oils. In prior analyses of essential oils, Nematollahi et al. (2018a) found the most prevalent VOCs (emitted from more than $70 \%$ of 24 oils) were alpha-pinene, limonene, and acetone. Chiang et al. (2010) found the most prevalent ( $>40 \%$ of 5 oils) were limonene, eucalyptol, and camphor. Francis and Stusdal (2014) found the most prevalent ( $>70 \%$ of 3 oils) were beta-pinene, p-cymene, limonene, menthone, menthofuran, neomenthol, menthol, 4-terpineol, menthyl acetate, and $\beta$-bourbonene. Chiu et al. (2009) found the most prevalent ( $>60 \%$ of 5 oils) were limonene, eucalyptol, heneicosane, and alpha-terpineol. Thus, commonly emitted compounds were terpenes (e.g., limonene, beta-pinene, and alpha-pinene).

Despite this prior work, little information exists on the specific VOCs, including potentially hazardous compounds, emitted from commercially available essential oils that make therapeutic claims for health and well-being.

This study investigates VOCs emitted from 14 therapeutic essential oils, with four main objectives: (1) to analyze VOC emissions from individual essential oils with therapeutic claims, (2) to identify VOCs classified as potentially hazardous, (3) to determine the most prevalent VOCs and potentially hazardous VOCs, and (4) to compare differences between the VOCs emitted and the ingredients listed on essential oil labels, safety data sheets, and manufacturers' websites.

\section{Materials and Methods}

For this study, a set of 14 essential oils were analyzed, representing different brands and aromas, including lavender oil, eucalyptus oil, tea tree oil, geranium oil, peppermint oil, bergamot oil, orange oil, and oil blends. Essential oils were selected based on claims of therapeutic benefits, such as the following: alleviates symptoms of colds, coughs, and flus; provides calming and relaxing effects; eases tension, headaches, stress, anxiety, depression, and anger; offers a natural antiseptic; soothes irritated skin; and promotes peaceful sleep for both adults and babies. Essential oils in this study were purchased from aromatherapy stores, supermarkets, pharmacies, and organic stores, in both Australia and the United States. 
Headspace GC/MS was used to analyze VOCs emitted from the essential oils using a capillary column and an automated injection system. Details about the analytic methods are provided in Nematollahi et al. (2018b).

"Potentially hazardous VOCs were identified according to classifications of (i) hazardous air pollutants (HAPs), United States Environmental Protection Agency (EPA 2017), including carcinogenic HAPs (EPA 2018), (ii) Hazardous Chemical Information System (HCIS), Safe Work Australia (SWA 2020), and (iii) asthmagens, Association of Occupational and Environmental Clinics (AOEC 2020)" following Steinemann (et al. 2020). This classification approach, however, is not intended as an assessment of safety or hazard from use of the essential oils.

\section{Results and Discussion}

\section{VOCs emitted}

The VOCs emitted from this group of essential oils are summarized in Table 1. In this paper: "The term 'VOC occurrences' refers to the number of individual VOCs emitted from the products, such that each VOC occurrence represents a single volatile ingredient in a single product. The term 'VOC identities' refers to the number of distinctly named VOCs emitted from the products, such that each VOC identity represents a compound, according to name and CAS number, that occurs in one or more of the products" following Steinemann et al. (2020).

Among the 14 essential oils, 1,034 VOC occurrences, representing 378 VOC identities, were emitted. Each essential oil emitted between 40 to 116 VOCs. Detailed information about the VOCs emitted from each of the essential oils is provided in Supplementary Tables 1 and 2.

\section{Most prevalent VOCs}

Among the 14 essential oils, the most prevalent VOCs (in more than $90 \%$ of all essential oils) were acetaldehyde, alpha-phellandrene, alpha-pinene, camphene, limonene, methanol, terpinolene, 3-carene, acetone, beta-phellandrene, ethanol, and gamma-terpinene (Table 2).

\section{Potentially hazardous emissions}

All essential oils emitted at least 9 VOCs classified as potentially hazardous, and $50 \%$ of the oils emitted at least 20 potentially hazardous VOCs (Supplementary Table 1). Among the 14 essential oils, the most prevalent potentially hazardous VOCs (in more than $90 \%$ of all essential oils) were acetaldehyde, limonene, methanol, acetone, ethanol, and 3-carene (Table 3). To note, acetaldehyde, limonene and methanol were emitted from all of the essential oils.

\section{Regulatory classifications}

Among the 1,034 VOCs emitted, 251 VOC occurrences (60 VOC identities) are classified as potentially hazardous under one or more criteria (Table 3). Specifically, among the 251 VOCs: 225 VOCs (56 identities) are classified as potentially hazardous under Safe Work Australia (SWA 2020); 65 VOCs (11 identities) under California Proposition 65 (OEHHA 2020); 55 VOCs (8 identities) as Hazardous Air Pollutants (EPA 2017), including 15 VOCs (2 identities) as carcinogenic HAPs (EPA 2018); and 15 VOCs (2 identities) as asthmagens under the Association of Occupational and Environmental Clinics (AOEC 2020). 


\section{Listing of ingredients}

Among all the 1,034 VOC occurrences, only 9 VOCs were listed on any essential oil label, safety data sheet, or website. Thus, fewer than $1 \%$ of all identified VOCs were disclosed. Moreover, among all the 251 VOCs classified as potentially hazardous, only 1 VOC was listed, on a website. Thus, fewer than $1 \%$ of all identified potentially hazardous VOCs were disclosed.

\section{Conclusions}

This study analyzed VOC emissions from a variety of 14 essential oils that make therapeutic claims. The analyses found the oils collectively emitted 1,034 VOCs, representing 378 VOC identities. Among these emissions, 251 VOCs, representing 60 VOC identities, are classified as potentially hazardous. The most prevalent VOCs emitted (in all 14 oils) were acetaldehyde, alpha-phellandrene, alpha-pinene, camphene, limonene, methanol, and terpinolene. The most prevalent potentially hazardous VOCs emitted (in all 14 oils) were acetaldehyde, limonene, and methanol. Results from this study provide new information on volatile compounds emitted from therapeutic essential oils, which can help to improve public awareness about potential exposures and risks.

\section{Acknowledgments}

We thank the supporters of this study: the Clean Air and Urban Landscapes Hub, funded by the Australian Government’s National Environmental Science Program; and the Commonwealth Scientific and Industrial Research Organisation (CSIRO) Land and Water. We also thank the anonymous reviewers of this paper. 


\section{References}

Cavanagh HM, Wilkinson JM (2002) Biological activities of lavender essential oil. Phytotherapy research 16(4):301-8.

Chiang HM, Chiu HH, Lai YM, Chen CY, Chiang HL (2010) Carbonyl species characteristics during the evaporation of essential oils. Atmos Environ 44:2240-2247.

Chiu HH, Chiang HM, Lo CC, et al. (2009) Constituents of volatile organic compounds of evaporating essential oil. Atmos Environ 43:5743-5749.

(AOEC) Association of Occupational and Environmental Clinics 2020. Exposure Code List. Asthmagens. http://www.aoecdata.org/ (accessed July 2020)

(EPA) Environmental Protection Agency 2017. Initial List of Hazardous Air Pollutants with Modifications. https://www.epa.gov/haps/initial-list-hazardous-air-pollutants-modifications (accessed July 2020)

(EPA) Environmental Protection Agency 2018. Prioritized Chronic Dose-Response Values. Weight of evidence for carcinogenicity. https://www.epa.gov/sites/production/files/201405/documents/table1.pdf (accessed July 2020)

Francis GW, Stusdal TK (2014) Differential evaporation of aromatherapy mint oils. Air Qual Atmos Heal 7:481-487.

Huang HL, Tsai TJ, Hsu NY, Lee CC, Wu PC, Su HJ (2012) Effects of essential oils on the formation of formaldehyde and secondary organic aerosols in an aromatherapy environment. Build Environ 57:120-125.

Milhem SA, Verriele M, Nicolas M, Thevenet F (2020) Does the ubiquitous use of essential oil-based products promote indoor air quality? A critical literature review. Environmental Science and Pollution Research 11:1-47.

Nematollahi N, Kolev SD, Steinemann A (2018a) Volatile chemical emissions from essential oils. Air Quality, Atmosphere \& Health 11(8):949-54.

Nematollahi N, Doronila A, Mornane PJ, Duan A, Kolev SD, Steinemann A (2018b) Volatile Chemical Emissions from Fragranced Baby Products. Air Quality, Atmosphere \& Health 11:785-90. doi: 10.1007/s11869-018-0593-1

(OEHHA) The Proposition 65 List 2020. Chemicals that are known to cause cancer or birth defects or other reproductive harm. https://oehha.ca.gov/proposition-65/proposition-65-list

Safe Work Australia (SWA), Hazardous Chemical Information System (HCIS): Search Hazardous Chemicals, http://hcis.safeworkaustralia.gov.au/HazardousChemical, (accessed July 2020)

Sarkic A, Stappen I (2018) Essential oils and their single compounds in cosmetics-A critical review. Cosmetics 5(1):11.

Shaaban HA, El-Ghorab AH, Shibamoto T (2012) Bioactivity of essential oils and their volatile aroma components. Journal of Essential Oil Research 24(2):203-12.

Steinemann A, Nematollahi N, Rismanchi B, Goodman N, Kolev SD (2020) Pandemic products and volatile chemical emissions. Air Quality, Atmosphere \& Health (17 Aug) 
Table 1: VOCs emitted from the 14 essential oils *

Emitted Listed

(on product label, safety data sheet, or website)

\begin{tabular}{lllll}
\hline $\begin{array}{l}\text { Number of } \\
\text { essential oils }\end{array}$ & All emitted VOCs & $\begin{array}{l}\text { Potentially hazardous } \\
\text { VOCs }\end{array}$ & All listed VOCs & $\begin{array}{l}\text { Potentially hazardous } \\
\text { VOCs }\end{array}$ \\
\hline 14 & $\begin{array}{l}\text { 1,034 occurrences } \\
\text { 378 identities }\end{array}$ & $\begin{array}{l}\text { 251 occurrences } \\
\text { 60 identities }\end{array}$ & $\begin{array}{l}9 \text { occurrences } \\
\text { 8 identities }\end{array}$ & $\begin{array}{l}\text { 1 occurrence } \\
1 \text { identity }\end{array}$ \\
\hline
\end{tabular}

*"VOC occurrences" refers to the number of individual VOCs emitted from the essential oils.

"VOC identities" refers to the number of unique VOCs emitted from one or more of the essential oils. 
Table 2: Most prevalent VOCs emitted from the 14 essential oils

\begin{tabular}{lll}
\hline Compound & CAS \# & $\begin{array}{l}\text { Prevalence } \\
\text { (\# of Products) }\end{array}$ \\
\hline Acetaldehyde* & $75-07-0$ & 14 \\
alpha-Phellandrene & $99-83-2$ & 14 \\
alpha-Pinene & $80-56-8$ & 14 \\
Camphene & $79-92-5$ & 14 \\
Limonene* & $138-86-3$ & 14 \\
Methanol* & $67-56-1$ & 14 \\
Terpinolene & $586-62-9$ & 14 \\
3-Carene* & $13466-78-9$ & 13 \\
Acetone* & $67-64-1$ & 13 \\
beta-Phellandrene & $555-10-2$ & 13 \\
Ethanol* & $64-17-5$ & 13 \\
gamma-Terpinene & $99-85-4$ & 13 \\
alpha-Terpineol & $98-55-5$ & 12 \\
beta-Pinene & $127-91-3$ & 12 \\
Isovaleraldehyde & $590-86-3$ & 12 \\
Linalool & $78-70-6$ & 12 \\
beta-Myrcene* & $123-35-3$ & 11 \\
Isobutyraldehyde & $78-84-2$ & 11 \\
1,1-Dimethylallyl alcohol & $115-18-4$ & 10 \\
2-Methylfuran & $534-22-5$ & 10 \\
3-Methylfuran & $930-27-8$ & 10 \\
beta-trans-Ocimene & $3779-61-1$ & 10 \\
Toluene* & $108-88-3$ & 9 \\
Caryophyllene & $87-44-5$ & 10 \\
Eucalyptol & $470-82-6$ & 9 \\
Pentane* & $109-66-0$ & \\
\hline Classified as potentally & & 12 \\
\hline
\end{tabular}

*Classified as potentially hazardous 
Table 3: Potentially hazardous VOCs emitted from the 14 essential oils

\begin{tabular}{llllll}
\hline Compound & CAS \# & $\begin{array}{l}\text { Prevalence } \\
\text { (\# of Products) }\end{array}$ & SWA & Prop65 HAPs & Asthmagens
\end{tabular}

\begin{tabular}{|c|c|c|c|c|c|c|}
\hline Acetaldehyde** & $75-07-0$ & 14 & $\checkmark$ & $\checkmark$ & $\checkmark$ & \\
\hline Limonene & $138-86-3$ & 14 & $\checkmark$ & & & \\
\hline Methanol & $67-56-1$ & 14 & $\checkmark$ & $\checkmark$ & $\checkmark$ & \\
\hline Acetone & $67-64-1$ & 13 & $\checkmark$ & & & \\
\hline Ethanol & $64-17-5$ & 13 & $\checkmark$ & & & \\
\hline 3-Carene & $13466-78-9$ & 13 & & & & $\checkmark$ \\
\hline beta-Myrcene & $123-35-3$ & 11 & & $\checkmark$ & & \\
\hline Toluene & $108-88-3$ & 10 & $\checkmark$ & $\checkmark$ & $\checkmark$ & \\
\hline Pentane & $109-66-0$ & 9 & $\checkmark$ & & & \\
\hline 2-Methylpropan-1-ol & $78-83-1$ & 8 & $\checkmark$ & & & \\
\hline Butanone & $78-93-3$ & 8 & $\checkmark$ & & & \\
\hline Ethyl acetate & $141-78-6$ & 8 & $\checkmark$ & & & \\
\hline 2-Methyl-1-propene & $115-11-7$ & 7 & $\checkmark$ & & & \\
\hline Isopropyl alcohol & $67-63-0$ & 7 & $\checkmark$ & & & \\
\hline Methyl acetate & $79-20-9$ & 7 & $\checkmark$ & & & \\
\hline 1-Hexanol & $111-27-3$ & 6 & $\checkmark$ & & & \\
\hline Methyl isobutyl ketone & $108-10-1$ & 6 & $\checkmark$ & $\checkmark$ & $\checkmark$ & \\
\hline Propanal & $123-38-6$ & 6 & $\checkmark$ & & $\checkmark$ & \\
\hline Butyl butyrate & $109-21-7$ & 5 & $\checkmark$ & & & \\
\hline Methyl formate & $107-31-3$ & 5 & $\checkmark$ & & & \\
\hline Ethyl formate & $109-94-4$ & 4 & $\checkmark$ & & & \\
\hline Fenchyl alcohol & $1632-73-1$ & 4 & $\checkmark$ & & & \\
\hline 2,4-Dimethylhexane & $589-43-5$ & 3 & $\checkmark$ & & & \\
\hline 2-Methylpropyl ester & $110-19-0$ & 3 & $\checkmark$ & & & \\
\hline 3-Methylhexane & $589-34-4$ & 3 & $\checkmark$ & & & \\
\hline Butane & $106-97-8$ & 3 & $\checkmark$ & & & \\
\hline Butyl acetate & $123-86-4$ & 3 & $\checkmark$ & & & \\
\hline Hexane & $110-54-3$ & 3 & $\checkmark$ & $\checkmark$ & $\checkmark$ & \\
\hline Isopentane & $78-78-4$ & 3 & $\checkmark$ & & & \\
\hline (Z)-but-2-ene & $590-18-1$ & 2 & $\checkmark$ & & & \\
\hline 2-Butanol & $78-92-2$ & 2 & $\checkmark$ & & & \\
\hline 2-Butene & $107-01-7$ & 2 & $\checkmark$ & & & \\
\hline 2-Methylpentane & $107-83-5$ & 2 & $\checkmark$ & & & \\
\hline Isoamyl acetate & $123-92-2$ & 2 & $\checkmark$ & & & \\
\hline Isoprene (stabilised) & $78-79-5$ & 2 & $\checkmark$ & $\checkmark$ & & \\
\hline Tetracarbonylnickel & $13463-39-3$ & 2 & $\checkmark$ & $\checkmark$ & & $\checkmark$ \\
\hline (E)-citral & $141-27-5$ & 1 & $\checkmark$ & & & \\
\hline (R)-(-)-2-butanol & 14898-79-4 & 1 & $\checkmark$ & & & \\
\hline 1-Heptanol & $111-70-6$ & 1 & $\checkmark$ & & & \\
\hline
\end{tabular}


1-Methoxy-2-propanol

1-Octanol

2,2-Dimethylbutane

2,3,4-Trimethylpentane

2-Methoxy-1-methylethyl

acetate

2-Methylbutyl acetate

3-Ethyl-3-methylpentane

3-Methylpentane

3-Pentanone

Acetaldehyde diethyl acetal

Benzene***

Butyl formate

Butyraldehyde

Furan

Isobutyl methacrylate

Isopentyl formate

Isopropyl propionate

Methyl isopropyl ketone

sec-Butyl acetate

(R)-(+)-pulegone

Dimethyl phthalate

\begin{tabular}{|c|c|c|c|c|}
\hline 107-98-2 & 1 & $\checkmark$ & & \\
\hline $111-87-5$ & 1 & $\checkmark$ & & \\
\hline $75-83-2$ & 1 & $\checkmark$ & & \\
\hline $565-75-3$ & 1 & $\checkmark$ & & \\
\hline $108-65-6$ & 1 & $\checkmark$ & & \\
\hline $624-41-9$ & 1 & $\checkmark$ & & \\
\hline 1067-08-9 & 1 & $\checkmark$ & & \\
\hline $96-14-0$ & 1 & $\checkmark$ & & \\
\hline $96-22-0$ & 1 & $\checkmark$ & & \\
\hline $105-57-7$ & 1 & $\checkmark$ & & \\
\hline $71-43-2$ & 1 & $\checkmark$ & $\checkmark$ & $\checkmark$ \\
\hline 592-84-7 & 1 & $\checkmark$ & & \\
\hline $123-72-8$ & 1 & $\checkmark$ & & \\
\hline $110-00-9$ & 1 & $\checkmark$ & $\checkmark$ & \\
\hline $97-86-9$ & 1 & $\checkmark$ & & \\
\hline $110-45-2$ & 1 & $\checkmark$ & & \\
\hline $637-78-5$ & 1 & $\checkmark$ & & \\
\hline 563-80-4 & 1 & $\checkmark$ & & \\
\hline $105-46-4$ & 1 & $\checkmark$ & & \\
\hline 89-82-7 & 1 & & $\checkmark$ & \\
\hline $131-11-3$ & 1 & & & $\checkmark$ \\
\hline
\end{tabular}

SWA: Safe Work Australia (SWA) contains a Hazardous Chemical Information System ("HCIS") with a database of chemicals and hazard classifications (SWA 2020).

Prop65: California Proposition 65 (OEHHA 2020)

HAPs: Hazardous Air Pollutants (HAPs), United States Environmental Protection Agency (EPA 2017), including carcinogenic HAPs (EPA 2018)

**Classified as possibly carcinogenic (2B) (EPA 2018)

*** Classified as carcinogenic (1) (EPA 2018)

Asthmagens: Association of Occupational and Environmental Clinics (AOEC 2020) 
Supplementary Table 1: VOC occurrences among essential oils $(n=14)$

\section{Lavender essential oil}

\begin{tabular}{ll}
\hline Compounds & CAS\# \\
\hline Linalool & $78-70-6$ \\
\hline Limonene* & $138-86-3$ \\
\hline Linalyl acetate & $115-95-7$ \\
\hline (Z)-beta-ocimene & $3338-55-4$ \\
\hline 3-Octanone & $106-68-3$ \\
\hline Acetone* & $67-64-1$ \\
\hline beta-Myrcene* & $123-35-3$ \\
\hline (-)-Terpinen-4-ol & $20126-76-5$ \\
\hline Hexyl methyl ether & $4747-07-3$ \\
\hline alpha-Pinene & $80-56-8$ \\
\hline Isovaleraldehyde & $590-86-3$ \\
\hline Methanol* & $67-56-1$ \\
\hline Hexyl acetate & $142-92-7$ \\
\hline Lavandulyl acetate & $25905-14-0$ \\
\hline Camphene & $79-92-5$ \\
\hline 1-Octen-3-yl-acetate & $2442-10-6$ \\
\hline alpha-Phellandrene & $99-83-2$ \\
\hline beta-Phellandrene & $555-10-2$ \\
\hline Caryophyllene & $87-44-5$ \\
\hline Isobutyraldehyde & $78-84-2$ \\
\hline 3-Carene* & $13466-78-9$ \\
\hline 2-Methylbutyraldehyde & $96-17-3$ \\
\hline 3-Methylfuran & $930-27-8$ \\
\hline Acetaldehyde* & $75-07-0$ \\
\hline 2-Methyl-1-propene* & $115-11-7$ \\
\hline 2,3-Pentadiene & $591-96-8$ \\
\hline gamma-Terpinene & $99-85-4$ \\
\hline o-Cymene & $527-84-4$ \\
\hline Ethanol* & $64-17-5$ \\
\hline alpha-Terpineol & $98-55-5$ \\
\hline 2-Methyl-1-butene & $563-46-2$ \\
\hline Butyl butyrate* & $109-21-7$ \\
\hline 2-Methylfuran & $534-22-5$ \\
\hline 1-Octen-3-ol & $3391-86-4$ \\
\hline Neryl acetate & $141-12-8$ \\
\hline 1,1-Dimethylallyl alcohol & $115-18-4$ \\
\hline Butyl methyl ether & $628-28-4$ \\
\hline Hexyl butyrate & $2639-63-6$ \\
\hline Borneol & $10385-78-1$ \\
\hline Terpinolene & $586-62-9$ \\
\hline Butyl acetate* & $123-86-4$ \\
\hline beta-trans-Ocimene & $3779-61-1$ \\
\hline 3-Octanol & $589-98-0$ \\
\hline & \\
\hline
\end{tabular}




\begin{tabular}{ll}
\hline trans- $\beta$-Farnesene & $18794-84-8$ \\
\hline 2-Ethylfuran & $3208-16-0$ \\
\hline (+)-Camphor & $464-49-3$ \\
\hline Sabinene & $3387-41-5$ \\
\hline (4E,6Z)-2,6-dimethylocta-2,4,6-triene & $7216-56-0$ \\
\hline Toluene* & $108-88-3$ \\
\hline trans-2-Methyl-1,3-pentadiene & $926-54-5$ \\
\hline Dimethyl sulfide & $75-18-3$ \\
\hline cis-1,2-Dimethylcyclopropane & $930-18-7$ \\
\hline (E)-linalool oxide (furanoid) & $34995-77-2$ \\
\hline 1-Hexanol* & $111-27-3$ \\
\hline Pentane* & $109-66-0$ \\
\hline alpha-Santalene & $512-61-8$ \\
\hline Geranyl acetate & $105-87-3$ \\
\hline Isopropyl alcohol* & $67-63-0$ \\
\hline Methyl acetate* & $79-20-9$ \\
\hline 4-Isopropyl-2-cyclohexenone & $500-02-7$ \\
\hline Bornyl acetate & $76-49-3$ \\
\hline Isoamyl alcohol & $123-51-3$ \\
\hline Iso-propylcyclopropane & $3638-35-5$ \\
\hline Butanone* & $78-93-3$ \\
\hline cis-3-Hexene-1-ol & $928-96-1$ \\
\hline 3,7-dimethyl-2,6-octadienyl isobutyrate & $2345-26-8$ \\
\hline Hexyl isobutyrate & $2349-07-7$ \\
\hline (E)-sabinene hydrate & $17699-16-0$ \\
\hline 2-Octynoic acid & $5663-96-7$ \\
\hline Propanal* & $123-38-6$ \\
\hline 8-Isopropyl-1-methyl-5-methylene-1,6- & $23986-74-5$ \\
\hline cyclodecadiene & $1487-99-6$ \\
\hline 2,7-Dimethyloxepine & $2445-76-3$ \\
\hline Hexyl propionate & $97-87-0$ \\
\hline Butyl isobutyrate & $78-83-1$ \\
\hline 2-Methylpropan-1-ol* & $16491-36-4$ \\
\hline cis-3-Hexenyl butyrate & $34713-94-5$ \\
\hline 2-Methyl-1-butanol & $108-10-1$ \\
\hline Methyl isobutyl ketone* & $78-85-3$ \\
\hline Methacrolein & $19883-29-5$ \\
\hline (E,E)-1,3,5-Undecatriene & $15537-55-0$ \\
\hline (Z)-sabinene hydrate & $625-86-5$ \\
\hline 2,5-Dimethylfuran & $17699-05-7$ \\
\hline alpha-Bergamotene & $141-78-6$ \\
\hline Ethyl acetate* & $1502-24-5$ \\
\hline 2,3-Dimethylcyclohexan-1-ol & $104-51-8$ \\
\hline Butylbenzene & $590-01-2$ \\
\hline Butyl propionate & $70220-06-3$ \\
\hline (Z)-1-methoxyhex-3-ene & $691-38-3$ \\
\hline cis-4-Methyl-2-Pentene & $123-72-8$ \\
\hline Butyraldehyde* & \\
\hline & \\
\hline
\end{tabular}




\begin{tabular}{ll}
\hline 2,2,3-Trimethylhexane & $16747-25-4$ \\
\hline gamma-Muurolene & $30021-74-0$ \\
\hline Nerol oxide & $1786-08-9$ \\
\hline 3,4-Dimethyl-2,4,6-octatriene & $57396-75-5$ \\
\hline cis-Hexatriene & $2612-46-6$ \\
\hline 3-Hexanone & $589-38-8$ \\
\hline Hexyl 2-methylbutanoate & $10032-15-2$ \\
\hline Phenyl butyrate & $4346-18-3$ \\
\hline alpha-Humulene & $6753-98-6$ \\
\hline 3,4,4-Trimethyl-2-cyclopenten-1-one & $30434-65-2$ \\
\hline 1-Butyne & $107-00-6$ \\
\hline Cyclobutane & $287-23-0$ \\
\hline p, $\alpha$-Dimethylstyrene & $1195-32-0$ \\
\hline Tetracarbonylnickel & $13463-39-3$ \\
\hline 1-Pentene & $109-67-1$ \\
\hline
\end{tabular}

*Classified as potentially hazardous 


\section{Geranium essential oil}

\begin{tabular}{ll}
\hline Compounds & CAS\# \\
\hline alpha-Pinene & $80-56-8$ \\
\hline Citronellyl formate & $105-85-1$ \\
\hline Menthone & $10458-14-7$ \\
\hline Linalool & $78-70-6$ \\
\hline B-Citronellol & $106-22-9$ \\
\hline Acetone* & $67-64-1$ \\
\hline Geranyl acetate & $105-87-3$ \\
\hline Aristolene & $6831-16-9$ \\
\hline 7-Methyl-1,6-octadiene & $42152-47-6$ \\
\hline Geraniol & $106-24-1$ \\
\hline (+)-Isomenthone & $1196-31-2$ \\
\hline Limonene* & $138-86-3$ \\
\hline Isoprene (stabilised)* & $78-79-5$ \\
\hline Dimethyl sulfide & $75-18-3$ \\
\hline 3-Methyl-1-cyclopentene & $1120-62-3$ \\
\hline beta-Myrcene* & $123-35-3$ \\
\hline 3-Methylfuran & $930-27-8$ \\
\hline 2-Methylfuran & $534-22-5$ \\
\hline Methyl formate* & $107-31-3$ \\
\hline (Z)-rose oxide & $16409-43-1$ \\
\hline alpha-Phellandrene & $99-83-2$ \\
\hline beta-Bourbonene & $5208-59-3$ \\
\hline Caryophyllene & $87-44-5$ \\
\hline (Z)-beta-ocimene & $3338-55-4$ \\
\hline 2,5-Dimethylhex-5-en-3-yn-2-ol & $2696-26-6$ \\
\hline 2-Pentanone & $107-87-9$ \\
\hline o-Cymene & $527-84-4$ \\
\hline Isobutyraldehyde & $78-84-2$ \\
\hline beta-Phellandrene & $555-10-2$ \\
\hline alpha-Pinene oxide & $1686-14-2$ \\
\hline trans-2-Methyl-1,3-pentadiene & $926-54-5$ \\
\hline alpha-Copaene & $3856-25-5$ \\
\hline Cyclofenchene & $488-97-1$ \\
\hline Ethyl formate* & $109-94-4$ \\
\hline cis-4-Methyl-2-Pentene & $691-38-3$ \\
\hline Isovaleraldehyde & $590-86-3$ \\
\hline Butyl propionate & $590-01-2$ \\
\hline Neryl acetate & $141-12-8$ \\
\hline 3-Allylcyclohexene & $15232-95-8$ \\
\hline Acetaldehyde* & $75-07-0$ \\
\hline 2,4-Dimethylhexane* & $589-43-5$ \\
\hline Pentane* & $109-66-0$ \\
\hline 8-Isopropyl-1-methyl-5-methylene-1,6- & $23986-74-5$ \\
\hline cyclodecadiene & $7392-19-0$ \\
\hline 2,2,6-Trimethyl-6-vinyltetrahydropyran & \\
\hline & \\
\hline
\end{tabular}




\begin{tabular}{ll}
\hline 5-Methyl-1,4-hexadiene & $763-88-2$ \\
\hline Isopentyl formate* & $110-45-2$ \\
\hline 3-Carene* & $13466-78-9$ \\
\hline 1-Cyclopropyl-1-pentanol & $4379-16-2$ \\
\hline Toluene* & $108-88-3$ \\
\hline 6-Methyl-5-hepten-2-one & $110-93-0$ \\
\hline 2,7-Dimethyloxepine & $1487-99-6$ \\
\hline 2-Methyl-1-propene* & $115-11-7$ \\
\hline cis-3,7-Dimethyl-2,6-octadien-1-ol & $106-25-2$ \\
\hline 1-Methyl-3-isopropylcyclopentane & $53771-88-3$ \\
\hline alpha-Gurjunene & $489-40-7$ \\
\hline Methanol* & $67-56-1$ \\
\hline 3-Undecyne & $60212-30-8$ \\
\hline 2,6-Dimethyl-3-heptene & $2738-18-3$ \\
\hline (E)-linalool oxide (furanoid) & $34995-77-2$ \\
\hline Vinylcyclohexane & $695-12-5$ \\
\hline trans-3,4-Dimethyl-2-pentene & $4914-92-5$ \\
\hline Acetylcyclohexane & $823-76-7$ \\
\hline 2-(1,1-Dimethyl-2-propenyl)-1,1- & $81051-15-2$ \\
\hline dimethylcyclopropane & \\
\hline beta-Cadinene & $523-47-7$ \\
\hline Terpinolene & $586-62-9$ \\
\hline Geranyl propionate & $105-90-8$ \\
\hline Ethanol* & $64-17-5$ \\
\hline 3-Methyl-1-pentanol & $589-35-5$ \\
\hline Methyl isobutyl ketone* & $108-10-1$ \\
\hline Acetaldol & $107-89-1$ \\
\hline alpha-Terpineol & $98-55-5$ \\
\hline 1-Methyl-3-propylcyclooctane & $255885-37-1$ \\
\hline alpha-Bergamotene & $17699-05-7$ \\
\hline alpha-Terpinene & $99-86-5$ \\
\hline Isoamyl alcohol & $123-51-3$ \\
\hline Camphene & $79-92-5$ \\
\hline Citronellyl acetate & $150-84-5$ \\
\hline 3-Methylcrotonaldehyde & $107-86-8$ \\
\hline Butanone* & $78-93-3$ \\
\hline Carane, 4,5-epoxy-, trans & $6909-20-2$ \\
\hline Isopropylidenecyclohexane & $5749-72-4$ \\
\hline 2,5-Dimethylfuran & $625-86-5$ \\
\hline D,L-Menthol & $15356-70-4$ \\
\hline alpha-Humulene & $6753-98-6$ \\
\hline Butyl formate* & $592-84-7$ \\
\hline 1,2,3-Trimethylcyclopentene & $473-91-6$ \\
\hline Isopentane* & $78-78-4$ \\
\hline (+)-Sativene & $3650-28-0$ \\
\hline 3-Methyl-2-cyclopenten-1-one & $2758-18-1$ \\
\hline 2-Methyl-2-butene & $513-35-9$ \\
\hline Hexane* & $110-54-3$ \\
\hline & \\
\hline
\end{tabular}




\begin{tabular}{|c|c|}
\hline Geranyl butyrate & $106-29-6$ \\
\hline Citronellyl propionate & $141-14-0$ \\
\hline Phenethyl butyrate & $103-52-6$ \\
\hline (Z)-linalool oxide (furanoid) & 5989-33-3 \\
\hline$(-)-\alpha$-Cubebene & 17699-14-8 \\
\hline cis-3-Hexene-1-ol & $928-96-1$ \\
\hline 2-Bromocyclooctanone & $39261-18-2$ \\
\hline Bicyclo[4.1.0]hept-2-ene & $2566-57-6$ \\
\hline Myrtanyl acetate & 29021-36-1 \\
\hline gamma-Terpinene & $99-85-4$ \\
\hline 2-Methylpropan-1-ol* & 78-83-1 \\
\hline 1,3-Cyclohexadiene & $592-57-4$ \\
\hline Citronellyl butyrate & $141-16-2$ \\
\hline 1-Acetyl-2-methyl-1-cyclopentene & $3168-90-9$ \\
\hline Hexyl formate & $629-33-4$ \\
\hline Allyl nonanoate & $7493-72-3$ \\
\hline$\beta$-Selinene & $17066-67-0$ \\
\hline Perillen & $539-52-6$ \\
\hline ( \pm )-2-Methyl-1-butanol & $137-32-6$ \\
\hline Propanal* & $123-38-6$ \\
\hline$( \pm)$-Citronellal & $106-23-0$ \\
\hline 2-Methylpentane* & $107-83-5$ \\
\hline 1-Acetyl-1-cyclohexene & 932-66-1 \\
\hline 1,3-Dimethyl cyclohexene & $2808-76-6$ \\
\hline (4E)-4-Hexenyl acetate & 72237-36-6 \\
\hline
\end{tabular}




\section{Bergamot essential oil}

\begin{tabular}{ll}
\hline Compounds & CAS\# \\
\hline Limonene* & $138-86-3$ \\
\hline beta-Pinene & $127-91-3$ \\
\hline gamma-Terpinene & $99-85-4$ \\
\hline Linalyl acetate & $115-95-7$ \\
\hline alpha-Pinene & $80-56-8$ \\
\hline Linalool & $78-70-6$ \\
\hline beta-Phellandrene & $555-10-2$ \\
\hline Vinyl methacrylate & $4245-37-8$ \\
\hline alpha-Thujene & $2867-05-2$ \\
\hline 2,4,6-Trimethyl-2,4,6-triphenyl-1,3,5,2,4,6- & $546-45-2$ \\
trioxatrisilinane & \\
\hline Terpinolene & $586-62-9$ \\
\hline beta-trans-Ocimene & $3779-61-1$ \\
\hline alpha-Terpinene & $99-86-5$ \\
\hline beta-Thujene & $28634-89-1$ \\
\hline Camphene & $79-92-5$ \\
\hline Ethyl acetate* & $141-78-6$ \\
\hline Acetone* & $67-64-1$ \\
\hline Lavandulyl acetate & $25905-14-0$ \\
\hline alpha-Phellandrene & $99-83-2$ \\
\hline Ethanol* & $64-17-5$ \\
\hline Isopropyl alcohol* & $67-63-0$ \\
\hline alpha-Terpineol & $98-55-5$ \\
\hline (E)-sabinene hydrate & $17699-16-0$ \\
\hline Octanal & $124-13-0$ \\
\hline Neryl acetate & $141-12-8$ \\
\hline Octyl acetate & $112-14-1$ \\
\hline (E)-citral* & $141-27-5$ \\
\hline Caryophyllene & $87-44-5$ \\
\hline 6-Methyl-5-hepten-2-one & $110-93-0$ \\
\hline alpha-Bergamotene & $17699-05-7$ \\
\hline Nonanal & $124-19-6$ \\
\hline Acetaldehyde* & $75-07-0$ \\
\hline (Z)-sabinene hydrate & $15537-55-0$ \\
\hline 4-Terpineol & $562-74-3$ \\
\hline Decanal & $112-31-2$ \\
\hline Methanol* & $67-56-1$ \\
\hline 3-Methylhexane* & $589-34-4$ \\
\hline beta-Bisabolene & $495-61-4$ \\
\hline 1,1-Dimethylallyl alcohol & $115-18-4$ \\
\hline Hexanal & $66-25-1$ \\
\hline Classifis as pol &
\end{tabular}

*Classified as potentially hazardous 


\section{Tea tree essential oil}

\begin{tabular}{ll}
\hline Compounds & CAS\# \\
\hline Acetone* & $67-64-1$ \\
\hline alpha-Pinene & $80-56-8$ \\
\hline beta-Pinene & $127-91-3$ \\
\hline beta-Myrcene* & $123-35-3$ \\
\hline Limonene* & $138-86-3$ \\
\hline beta-Phellandrene & $555-10-2$ \\
\hline 4-Terpineol & $562-74-3$ \\
\hline gamma-Terpinene & $99-85-4$ \\
\hline 4-Carene & $29050-33-7$ \\
\hline (S)-cis-Verbenol & $18881-04-4$ \\
\hline Butyl butyrate* & $109-21-7$ \\
\hline 3-Methylfuran & $930-27-8$ \\
\hline Eucalyptol & $470-82-6$ \\
\hline alpha-Phellandrene & $99-83-2$ \\
\hline Ocimenol & $5986-38-9$ \\
\hline Hexyl butyrate & $2639-63-6$ \\
\hline 2-Methylfuran & $534-22-5$ \\
\hline Alloaromadendrene & $25246-27-9$ \\
\hline (+)-Ledene & $21747-46-6$ \\
\hline delta-Cadinene & $483-76-1$ \\
\hline Butane* & $106-97-8$ \\
\hline Acetaldehyde* & $75-07-0$ \\
\hline alpha-Gurjunene & $489-40-7$ \\
\hline Ethyl acetate* & $141-78-6$ \\
\hline 3,4,4-Trimethyl-2-cyclopenten-1-one & $30434-65-2$ \\
\hline 2-(1,1-Dimethyl-2-propenyl)-1,1- & $81051-15-2$ \\
\hline dimethylcyclopropane & \\
\hline Methanol* & $67-56-1$ \\
\hline Diethyl phthalate & $84-66-2$ \\
\hline Ethanol* & $64-17-5$ \\
\hline Citronellyl acetate & $150-84-5$ \\
\hline Toluene* & $108-88-3$ \\
\hline Methyl 4-methylvalerate & $2412-80-8$ \\
\hline alpha-Thujene & $2867-05-2$ \\
\hline Dimethyl phthalate* & $131-11-3$ \\
\hline Methyl (S)-2-methylbutanoate & $53955-81-0$ \\
\hline trans-2-Hexen-1-al & $6728-26-3$ \\
\hline 2,6-Dimethylhepta-1,5-diene & $6709-39-3$ \\
\hline 3-Carene* & $13466-78-9$ \\
\hline Methyl formate* & $107-31-3$ \\
\hline cis-1,2-Dimethylcyclopropane & $930-18-7$ \\
\hline Methyl 3-methylvalerate & $2177-78-8$ \\
\hline m-Cymene & $535-77-3$ \\
\hline beta-trans-Ocimene & $3779-61-1$ \\
\hline 2-Methyl-2-(4-methylpent-3-en-1- & $98678-70-7$ \\
\hline & \\
\hline
\end{tabular}




\begin{tabular}{ll}
\hline yl)cyclopropylmethanol & \\
\hline 2-Hexyne & $764-35-2$ \\
\hline Camphene & $79-92-5$ \\
\hline 3,5-Heptadienal, 2-ethylidene-6-methyl- & $99172-18-6$ \\
\hline 2-(2-Methyl-1-propenyl)bicyclo[2.2.1]heptane & $61142-27-6$ \\
\hline (Z)-rose oxide & $16409-43-1$ \\
\hline trans-2-Methyl-1,3-pentadiene & $926-54-5$ \\
\hline Furan* & $110-00-9$ \\
\hline Isovaleraldehyde & $590-86-3$ \\
\hline 3-Ethyl-2,2-dimethyloxirane & $1192-22-9$ \\
\hline 2,3,5-Trimethyl-1,3-hexadiene & $61142-34-5$ \\
\hline Terpinolene & $586-62-9$ \\
\hline Isobutyraldehyde & $78-84-2$ \\
\hline cis-4-Methyl-2-Pentene & $691-38-3$ \\
\hline sec-Butyl acetate* & $105-46-4$ \\
\hline (E)-linalool oxide (furanoid) & $34995-77-2$ \\
\hline Pentane* & $109-66-0$ \\
\hline 2-Methylbutyraldehyde & $96-17-3$ \\
\hline 2-Ethylfuran & $3208-16-0$ \\
\hline 1,1,2-Trimethylcyclopentane & $4259-00-1$ \\
\hline 3-Octyne & $15232-76-5$ \\
\hline Isopulegol & $7786-67-6$ \\
\hline Ethyl formate* & $109-94-4$ \\
\hline 2-Methoxy-1-methylethyl acetate* & $108-65-6$ \\
\hline 3-Methylcrotonaldehyde & $107-86-8$ \\
\hline 1-hydroxy-2-nitrocyclohexane-1-carboxylic acid & $149695-77-2$ \\
\hline Perillen & $539-52-6$ \\
\hline 2-Methyl-1-propene* & $115-11-7$ \\
\hline Isopropyl propionate* & $637-78-5$ \\
\hline Ethyl butyrate & $105-54-4$ \\
\hline Hexanal & $66-25-1$ \\
\hline Methyl valerate & $624-24-8$ \\
\hline 3-Methylcyclopentanol & $18729-48-1$ \\
\hline 4-Hexen-1-ol, acetate & $72237-36-6$ \\
\hline Lavandulyl acetate & $25905-14-0$ \\
\hline Isopropyl butyrate & $638-11-9$ \\
\hline Methyl 3-methyl-2-butenoate & $924-50-5$ \\
\hline *Classified as potentially hazardous & \\
& \\
\hline
\end{tabular}




\section{Eucalyptus essential oil}

\begin{tabular}{ll}
\hline Compounds & CAS\# \\
\hline Eucalyptol & $470-82-6$ \\
\hline alpha-Pinene & $80-56-8$ \\
\hline Limonene* & $138-86-3$ \\
\hline m-Cymene & $535-77-3$ \\
\hline beta-Pinene & $127-91-3$ \\
\hline gamma-Terpinene & $99-85-4$ \\
\hline 3-Carene* & $13466-78-9$ \\
\hline alpha-Thujene & $2867-05-2$ \\
\hline alpha-Terpineol & $98-55-5$ \\
\hline Ethanol* & $64-17-5$ \\
\hline beta-Myrcene* & $123-35-3$ \\
\hline 4-Carene & $29050-33-7$ \\
\hline alpha-Phellandrene & $99-83-2$ \\
\hline Camphene & $79-92-5$ \\
\hline 4-Terpineol & $562-74-3$ \\
\hline Acetone* & $67-64-1$ \\
\hline beta-Phellandrene & $555-10-2$ \\
\hline Isovaleraldehyde & $590-86-3$ \\
\hline Linalyl isobutyrate & $78-35-3$ \\
\hline (+)-Camphene & $5794-03-6$ \\
\hline Isoamyl alcohol & $123-51-3$ \\
\hline 2,4-Thujadiene & $36262-09-6$ \\
\hline Terpinolene & $586-62-9$ \\
\hline Methanol* & $67-56-1$ \\
\hline Isoamyl acetate* & $123-92-2$ \\
\hline 2-Methylbutyl acetate* & $624-41-9$ \\
\hline 2-Methyl-1-propene* & $115-11-7$ \\
\hline 1,4-Pentadiene & $591-93-5$ \\
\hline Isopropyl alcohol* & $67-63-0$ \\
\hline Iso-propylcyclopropane & $3638-35-5$ \\
\hline Isobutyraldehyde & $78-84-2$ \\
\hline 2,3-Dimethyl-1-butene & $563-78-0$ \\
\hline 1,1-Dimethylallyl alcohol & $115-18-4$ \\
\hline 2-Methylpropan-1-ol* & $78-83-1$ \\
\hline 2-Pentanone & $107-87-9$ \\
\hline Toluene* & $108-88-3$ \\
\hline 6-Bromocycloocta-1,4-diene & $23359-89-9$ \\
\hline 2,4-Dimethyl-2,4-heptadiene & $74421-05-9$ \\
\hline 2-Isopropenyltoluene & $7399-49-7$ \\
\hline 4-Isopropyl-2-cyclohexenone & $500-02-7$ \\
\hline Acetaldehyde* & $75-07-0$ \\
\hline 2-Methyl-1-butene & $563-46-2$ \\
\hline Methacrolein & $78-85-3$ \\
\hline 2-Methylfuran & $534-22-5$ \\
\hline trans-2-trans-4-Hexadiene & $6108-61-8$ \\
\hline & \\
\hline
\end{tabular}




\begin{tabular}{ll}
\hline Methyl isobutyl ketone* & $108-10-1$ \\
\hline 2-Methylpropyl ester* & $110-19-0$ \\
\hline Cyclopentene,4-ethenyl-1,5,5-trimethyl & $1727-69-1$ \\
\hline (-)-trans-Pinocarveol & $547-61-5$ \\
\hline *Classified as potentially hazardous &
\end{tabular}




\section{Eucalyptus essential oil}

\begin{tabular}{ll}
\hline Compounds & CAS\# \\
\hline Eucalyptol & $470-82-6$ \\
\hline Limonene* & $138-86-3$ \\
\hline alpha-Pinene & $80-56-8$ \\
\hline gamma-Terpinene & $99-85-4$ \\
\hline m-Cymene & $535-77-3$ \\
\hline 3-Carene* & $13466-78-9$ \\
\hline beta-Pinene & $127-91-3$ \\
\hline alpha-Thujene & $2867-05-2$ \\
\hline beta-Myrcene* & $123-35-3$ \\
\hline 4-Carene & $29050-33-7$ \\
\hline alpha-Terpineol & $98-55-5$ \\
\hline Terpinolene & $586-62-9$ \\
\hline Isovaleraldehyde & $590-86-3$ \\
\hline Ethanol* & $64-17-5$ \\
\hline 4-Terpineol & $562-74-3$ \\
\hline beta-trans-Ocimene & $3779-61-1$ \\
\hline Acetone* & $67-64-1$ \\
\hline Pentylidenecyclopentane & $53366-55-5$ \\
\hline alpha-Pinene oxide & $1686-14-2$ \\
\hline Linalool & $78-70-6$ \\
\hline Isoamyl alcohol & $123-51-3$ \\
\hline alpha-Phellandrene & $99-83-2$ \\
\hline Methanol* & $67-56-1$ \\
\hline Camphene & $79-92-5$ \\
\hline 2-Isopropenyltoluene & $7399-49-7$ \\
\hline (-)-trans-Pinocarveol & $547-61-5$ \\
\hline Linalool oxide & $1365-19-1$ \\
\hline (4E,6Z)-2,6-dimethylocta-2,4,6-triene & $7216-56-0$ \\
\hline 2-Methyl-1-propene* & $115-11-7$ \\
\hline Acetaldehyde* & $75-07-0$ \\
\hline Isobutyraldehyde & $78-84-2$ \\
\hline 2,3-Dimethyl-1-butene & $563-78-0$ \\
\hline 2-Methylpropan-1-ol* & $78-83-1$ \\
\hline Methyl isopropyl ketone* & $563-80-4$ \\
\hline Methyl isobutyl ketone* & $108-10-1$ \\
\hline Toluene* & $108-88-3$ \\
\hline Isoamyl acetate* & $123-92-2$ \\
\hline Isobutyl butyrate & $539-90-2$ \\
\hline Butylbenzene & $104-51-8$ \\
\hline (E)-linalool oxide (furanoid) & $34995-77-2$ \\
\hline Fenchyl alcohol* & $1632-73-1$ \\
\hline Ocimene & $13877-91-3$ \\
\hline Methyl acetate* & $79-20-9$ \\
\hline *Cans & \\
\hline
\end{tabular}

*Classified as potentially hazardous 


\section{Peppermint essential oil}

\begin{tabular}{|c|c|}
\hline Compounds & CAS\# \\
\hline Menthone & $89-80-5$ \\
\hline Menthol & $1490-04-6$ \\
\hline Eucalyptol & $470-82-6$ \\
\hline Limonene* & $138-86-3$ \\
\hline beta-Pinene & $127-91-3$ \\
\hline alpha-Pinene & $80-56-8$ \\
\hline 4,5,6,7-Tetrahydro-3,6-dimethylbenzofuran & $494-90-6$ \\
\hline beta-Phellandrene & $555-10-2$ \\
\hline Dl-Menthyl acetate & $16409-45-3$ \\
\hline D,l-Menthol & $15356-70-4$ \\
\hline m-Cymene & $535-77-3$ \\
\hline Acetone* & $67-64-1$ \\
\hline 4-Carene & $29050-33-7$ \\
\hline gamma-Terpinene & $99-85-4$ \\
\hline Methanol* & $67-56-1$ \\
\hline 2-Isopropylidene-5-methylcyclohexanone & $15932-80-6$ \\
\hline Caryophyllene & $87-44-5$ \\
\hline Isovaleraldehyde & $590-86-3$ \\
\hline 2-Methylbutyraldehyde & $96-17-3$ \\
\hline alpha-Phellandrene & $99-83-2$ \\
\hline Camphene & $79-92-5$ \\
\hline 2,5-Diethyl tetrahydrofuran & $41239-48-9$ \\
\hline alpha-Thujene & $2867-05-2$ \\
\hline Butane* & $106-97-8$ \\
\hline (R)-(+)-pulegone* & $89-82-7$ \\
\hline Isobutyraldehyde & $78-84-2$ \\
\hline 2-Ethylfuran & $3208-16-0$ \\
\hline Ethanol* & $64-17-5$ \\
\hline alpha-Terpineol & $98-55-5$ \\
\hline 1-Methyl-4-isopropyl-1-cyclohexen-3-one & $89-81-6$ \\
\hline 3-Methylcyclohexanone & $591-24-2$ \\
\hline Terpinolene & $586-62-9$ \\
\hline 3-Carene* & $13466-78-9$ \\
\hline (Z)-sabinene hydrate & $15537-55-0$ \\
\hline Acetaldehyde* & $75-07-0$ \\
\hline Neryl acetate & $141-12-8$ \\
\hline Isoamyl alcohol & $123-51-3$ \\
\hline beta-Bourbonene & $5208-59-3$ \\
\hline ( \pm )-2-Methyl-1-butanol & $137-32-6$ \\
\hline Toluene* & $108-88-3$ \\
\hline Ethyl 2-methylbutyrate & 7452-79-1 \\
\hline 2,4-Thujadiene & $36262-09-6$ \\
\hline 2-Methylfuran & $534-22-5$ \\
\hline 2-Methylpropan-1-ol* & $78-83-1$ \\
\hline 8-Isopropyl-1-methyl-5-methylene-1,6- & $23986-74-5$ \\
\hline
\end{tabular}




\begin{tabular}{ll}
\hline cyclodecadiene & \\
\hline Linalool & $78-70-6$ \\
\hline 2-Methylbutyl 2-methylbutyrate & $2445-78-5$ \\
\hline 3-Methylfuran & $930-27-8$ \\
\hline Pentane* & $109-66-0$ \\
\hline Butanone* & $78-93-3$ \\
\hline 1,1-Dimethylallyl alcohol & $115-18-4$ \\
\hline Isopropyl alcohol* & $67-63-0$ \\
\hline Methyl acetate* & $79-20-9$ \\
\hline 4,4-Dimethyl octane & $15869-95-1$ \\
\hline Methyl formate* & $107-31-3$ \\
\hline 2,4-Dimethylhexane* & $589-43-5$ \\
\hline Amyl isovalerate & $25415-62-7$ \\
\hline Pentanal & $110-62-3$ \\
\hline Isopentane* & $78-78-4$ \\
\hline 1-Octen-3-ol & $3391-86-4$ \\
\hline alpha-Humulene & $6753-98-6$ \\
\hline 2-Butene* & $107-01-7$ \\
\hline (R)-(+)-3-methylcyclopentanone & $6672-30-6$ \\
\hline Methyl (S)-2-methylbutanoate & $53955-81-0$ \\
\hline Benzene* & $71-43-2$ \\
\hline
\end{tabular}

*Classified as potentially hazardous 


\section{Lavender essential oil}

\begin{tabular}{ll}
\hline Compounds & CAS\# \\
\hline Linalool & $78-70-6$ \\
\hline Linalyl butyrate & $78-36-4$ \\
\hline Eucalyptol & $470-82-6$ \\
\hline Limonene* & $138-86-3$ \\
\hline 3-Octanone & $106-68-3$ \\
\hline (-)-Terpinen-4-ol & $20126-76-5$ \\
\hline Hexyl methyl ether & $4747-07-3$ \\
\hline alpha-Pinene & $80-56-8$ \\
\hline Acetone* & $67-64-1$ \\
\hline beta-Myrcene* & $123-35-3$ \\
\hline Hexyl acetate & $142-92-7$ \\
\hline Camphor & $76-22-2$ \\
\hline 3-Carene* & $13466-78-9$ \\
\hline alpha-Phellandrene & $99-83-2$ \\
\hline Camphene & $79-92-5$ \\
\hline cis-1,2-Dimethylcyclopropane & $930-18-7$ \\
\hline Neryl acetate & $141-12-8$ \\
\hline Bicyclo[5.2.0]nonane, 2-methylene-4,8,8- & $242794-76-9$ \\
trimethyl-4-vinyl- & \\
\hline Methanol* & $67-56-1$ \\
\hline beta-Pinene & $127-91-3$ \\
\hline gamma-Terpinene & $99-85-4$ \\
\hline m-Cymene & $535-77-3$ \\
\hline 1-Octen-3-yl-acetate & $2442-10-6$ \\
\hline Ocimenol & $5986-38-9$ \\
\hline Isovaleraldehyde & $590-86-3$ \\
\hline 3-Octanol & $589-98-0$ \\
\hline beta-trans-Ocimene & $3779-61-1$ \\
\hline (-)-Borneol & $464-45-9$ \\
\hline beta-Phellandrene & $555-10-2$ \\
\hline Hexyl butyrate & $2639-63-6$ \\
\hline Isobutyraldehyde & $78-84-2$ \\
\hline Benzo(b)thiophene-1,1-dioxide & $825-44-5$ \\
\hline 3-Methylfuran & $930-27-8$ \\
\hline 2-Methylpropyl ester* & $110-19-0$ \\
\hline Butyl butyrate* & $109-21-7$ \\
\hline 2-Methylbutyraldehyde & $96-17-3$ \\
\hline Terpinolene & $586-62-9$ \\
\hline Butyl methyl ether & $628-28-4$ \\
\hline 2-Methyl-1-propene* & $115-11-7$ \\
\hline trans- $\beta-F a r n e s e n e ~$ & $18794-84-8$ \\
\hline Acetaldehyde* & $75-07-0$ \\
\hline Ethanol* & $64-17-5$ \\
\hline 2-Ethylfuran & $3208-16-0$ \\
\hline 2-Methylfuran & $534-22-5$ \\
\hline & \\
\hline
\end{tabular}




\begin{tabular}{ll}
\hline 1-Hexanol* & $111-27-3$ \\
\hline 4-Methyl-1,3-pentadiene & $926-56-7$ \\
\hline 1,1-Dimethylallyl alcohol & $115-18-4$ \\
\hline Linalool oxide & $1365-19-1$ \\
\hline Toluene* & $108-88-3$ \\
\hline trans-1,2-Dimethylcyclopropane & $2402-06-4$ \\
\hline 1-Octen-3-ol & $3391-86-4$ \\
\hline Pentane* & $109-66-0$ \\
\hline Methacrolein & $78-85-3$ \\
\hline 4,4,6,6-Tetramethylbicyclo[3.1.0]hex-2-ene & $19487-09-3$ \\
\hline Hexyl isobutyrate & $2349-07-7$ \\
\hline Isopropyl alcohol* & $67-63-0$ \\
\hline Butyl isobutyrate & $97-87-0$ \\
\hline (Z)-sabinene hydrate & $15537-55-0$ \\
\hline Geranyl acetate & $105-87-3$ \\
\hline alpha-Santalene & $512-61-8$ \\
\hline 1-Methyl-3-(2-methylcyclopropyl)cyclopropene & $61142-26-5$ \\
\hline 4-Isopropyl-2-cyclohexenone & $500-02-7$ \\
\hline Methyl acetate* & $79-20-9$ \\
\hline Iso-propylcyclopropane & $3638-35-5$ \\
\hline Methyl isobutyl ketone* & $108-10-1$ \\
\hline 2,3,4-Trimethylhexane & $921-47-1$ \\
\hline cis-3-Hexene-1-ol & $928-96-1$ \\
\hline 2,7-Dimethyloxepine & $1487-99-6$ \\
\hline Lavandulyl acetate & $25905-14-0$ \\
\hline 8-Isopropyl-1-methyl-5-methylene-1,6- & $23986-74-5$ \\
\hline cyclodecadiene & \\
\hline Methyl butyrate & $623-42-7$ \\
\hline 3-Hexanone & $589-38-8$ \\
\hline Butyl propionate & $590-01-2$ \\
\hline Hexyl propionate & $2445-76-3$ \\
\hline 1-Pentene & $109-67-1$ \\
\hline Propanal* & $123-38-6$ \\
\hline Butanone* & $78-93-3$ \\
\hline Ethyl acetate* & $141-78-6$ \\
\hline 2-Methylpropan-1-o** & $78-83-1$ \\
\hline cis-Hexatriene & $2612-46-6$ \\
\hline 2,5-Dimethylfuran & $625-86-5$ \\
\hline Clasfied po pol & \\
\hline
\end{tabular}

*Classified as potentially hazardous 


\section{Mix essential oil}

\begin{tabular}{ll}
\hline Compounds & CAS\# \\
\hline Eucalyptol & $470-82-6$ \\
\hline Propylene glycol & $57-55-6$ \\
\hline alpha-Pinene & $80-56-8$ \\
\hline Limonene* & $138-86-3$ \\
\hline beta-Pinene & $127-91-3$ \\
\hline gamma-Terpinene & $99-85-4$ \\
\hline m-Cymene & $535-77-3$ \\
\hline Camphene & $79-92-5$ \\
\hline Menthol & $1490-04-6$ \\
\hline 3-Carene* & $13466-78-9$ \\
\hline alpha-Thujene & $2867-05-2$ \\
\hline beta-Myrcene* & $123-35-3$ \\
\hline alpha-Terpineol & $98-55-5$ \\
\hline Camphor & $76-22-2$ \\
\hline 4-Carene & $29050-33-7$ \\
\hline alpha-Phellandrene & $99-83-2$ \\
\hline Methyl salicylate & $119-36-8$ \\
\hline gamma-Terpineol & $586-81-2$ \\
\hline Terpinolene & $586-62-9$ \\
\hline beta-trans-Ocimene & $3779-61-1$ \\
\hline beta-Terpineol & $138-87-4$ \\
\hline Pentylidenecyclopentane & $53366-55-5$ \\
\hline Caryophyllene & $87-44-5$ \\
\hline Borneol & $10385-78-1$ \\
\hline Dihydrocarveol & $619-01-2$ \\
\hline 1-Terpinenol & $586-82-3$ \\
\hline Bornyl acetate & $76-49-3$ \\
\hline (+)-Longifolene & $475-20-7$ \\
\hline Cyclofenchene & $488-97-1$ \\
\hline 2,4-Thujadiene & $36262-09-6$ \\
\hline beta-Phellandrene & $555-10-2$ \\
\hline 3-Undecyne & $60212-30-8$ \\
\hline Acetaldehyde* & $75-07-0$ \\
\hline 2-Methylpentane* & $107-83-5$ \\
\hline Bornylene & $464-17-5$ \\
\hline Linalool & $78-70-6$ \\
\hline Methanol* & $67-56-1$ \\
\hline 2,2-Dimethylbutane* & $75-83-2$ \\
\hline 3-Methylpentane* & $96-14-0$ \\
\hline Hexane* & $110-54-3$ \\
\hline Isovaleraldehyde & $590-86-3$ \\
\hline 1-Methoxy-2-propanol* & $107-98-2$ \\
\hline (Z)-7-Methyl-5-undecene & $74630-62-9$ \\
\hline 3,8-p-Menthadiene & $586-67-4$ \\
\hline (1R,3R,4S)-2,2,3-trimethylbicyclo[2.2.1]heptane & $20536-41-8$ \\
\hline & \\
\hline
\end{tabular}




\begin{tabular}{ll}
\hline 2-Isopropenyltoluene & $7399-49-7$ \\
\hline (+)-Camphor & $464-49-3$ \\
\hline alpha-Ylangene & $14912-44-8$ \\
\hline 1-Pentene & $109-67-1$ \\
\hline 2-Methyl-2-pentene & $625-27-4$ \\
\hline 3-Methylfuran & $930-27-8$ \\
\hline Fenchyl alcohol & $1632-73-1$ \\
\hline *Classified as potentially hazardous &
\end{tabular}




\section{Mix essential oil}

\begin{tabular}{ll}
\hline Compounds & CAS\# \\
\hline Ethanol* & $64-17-5$ \\
\hline Eucalyptol & $470-82-6$ \\
\hline Linalyl butyrate & $78-36-4$ \\
\hline Limonene* & $138-86-3$ \\
\hline Linalool & $78-70-6$ \\
\hline beta-Myrcene* & $123-35-3$ \\
\hline alpha-Pinene & $80-56-8$ \\
\hline 3-Octanone & $106-68-3$ \\
\hline Camphene & $79-92-5$ \\
\hline Caryophyllene & $87-44-5$ \\
\hline Hexyl methyl ether & $474-07-3$ \\
\hline beta-Pinene & $127-91-3$ \\
\hline Hexyl acetate & $142-92-7$ \\
\hline m-Cymene & $535-77-3$ \\
\hline alpha-Phellandrene & $99-83-2$ \\
\hline beta-trans-Ocimene & $3779-61-1$ \\
\hline gamma-Terpinene & $99-85-4$ \\
\hline 3-Carene* & $13466-78-9$ \\
\hline Camphor & $76-22-2$ \\
\hline Terpinolene & $586-62-9$ \\
\hline Hexyl butyrate & $2639-63-6$ \\
\hline Butyl butyrate* & $109-21-7$ \\
\hline beta-Phellandrene & $555-10-2$ \\
\hline Neryl acetate & $141-12-8$ \\
\hline 4-Terpineol & $562-74-3$ \\
\hline 1-Octen-3-yl-acetate & $2442-10-6$ \\
\hline Hexyl isobutyrate & $2349-07-7$ \\
\hline Acetone* & $67-64-1$ \\
\hline Butyl acetate* & $123-86-4$ \\
\hline Fenchyl alcohol* & $1632-73-1$ \\
\hline cis-1,2-Dimethylcyclopropane & $930-18-7$ \\
\hline Ethyl formate* & $109-94-4$ \\
\hline 3-Methylfuran & $930-27-8$ \\
\hline Ethyl acetate* & $141-78-6$ \\
\hline Acetaldehyde* & $75-07-0$ \\
\hline Acetaldehyde diethyl acetal* & $105-57-7$ \\
\hline 4,4,6,6-Tetramethylbicyclo[3.1.0]hex-2-ene & $19487-09-3$ \\
\hline (-)-Borneol & $464-45-9$ \\
\hline alpha-Terpineol & $98-55-5$ \\
\hline Lavandulyl acetate & $25905-14-0$ \\
\hline Geranyl acetate & $105-87-3$ \\
\hline 2-Methylfuran & $534-22-5$ \\
\hline Isovaleraldehyde & $590-86-3$ \\
\hline 2[2(2-Methoxyethoxy)ethoxy]ethanol acetate & $3610-27-3$ \\
\hline Toluene* & $108-88-3$ \\
\hline & \\
\hline
\end{tabular}




\begin{tabular}{ll}
\hline Butyl isobutyrate & $97-87-0$ \\
\hline Hexyl propionate & $2445-76-3$ \\
\hline (2E)-1-ethoxy-3,7-dimethylocta-2,6-diene & $22882-91-3$ \\
\hline Methanol* & $67-56-1$ \\
\hline Pentane* & $109-66-0$ \\
\hline trans-1,2-Dimethylcyclopropane & $2402-06-4$ \\
\hline Isobutyraldehyde & $78-84-2$ \\
\hline Butyl methyl ether & $628-28-4$ \\
\hline trans-2-trans-4-Hexadiene & $6108-61-8$ \\
\hline 1,1-Dimethylallyl alcohol & $115-18-4$ \\
\hline 2-Methylbutyraldehyde & $96-17-3$ \\
\hline 2-Ethylfuran & $3208-16-0$ \\
\hline 2,4-Dimethylhexane* & $589-43-5$ \\
\hline cis-3-Nonen-1-ol & $10340-23-5$ \\
\hline 1-Hexanol* & $111-27-3$ \\
\hline D,L-isobornyl acetate & $92618-89-8$ \\
\hline alpha-Santalene & $512-61-8$ \\
\hline (Z)-but-2-ene* & $590-18-1$ \\
\hline Hexane* & $110-54-3$ \\
\hline Methacrolein & $78-85-3$ \\
\hline 3-Octyl acetate & $4864-61-3$ \\
\hline *Classified as potentially hazardous &
\end{tabular}

*Classified as potentially hazardous 


\section{Mix essential oil}

\begin{tabular}{|c|c|}
\hline Compounds & CAS\# \\
\hline Limonene* & $138-86-3$ \\
\hline Linalool & $78-70-6$ \\
\hline Linalyl butyrate & $78-36-4$ \\
\hline (Z)-beta-ocimene & $3338-55-4$ \\
\hline beta-Myrcene* & $123-35-3$ \\
\hline alpha-Pinene & $80-56-8$ \\
\hline$(-)$-Terpinen-4-ol & 20126-76-5 \\
\hline Ethyl 2-methylcyclopropanecarboxylate & 20913-25-1 \\
\hline 3-Octanone & $106-68-3$ \\
\hline Acetone* & $67-64-1$ \\
\hline Neryl acetate & $141-12-8$ \\
\hline Hexyl acetate & $142-92-7$ \\
\hline Caryophyllene & $87-44-5$ \\
\hline 3-Carene* & $13466-78-9$ \\
\hline beta-Phellandrene & $555-10-2$ \\
\hline 1-Octen-3-yl-acetate & $2442-10-6$ \\
\hline Methanol* & $67-56-1$ \\
\hline Iso-amyl-senecioate & $56922-73-7$ \\
\hline alpha-Phellandrene & $99-83-2$ \\
\hline alpha-Terpineol & $98-55-5$ \\
\hline Camphene & $79-92-5$ \\
\hline Hexyl methyl ether & $4747-07-3$ \\
\hline Isobutyl butyrate & $539-90-2$ \\
\hline beta-Pinene & $127-91-3$ \\
\hline beta-trans-Ocimene & 3779-61-1 \\
\hline Isovaleraldehyde & $590-86-3$ \\
\hline$(-)$-Borneol & $464-45-9$ \\
\hline Ethanol* & $64-17-5$ \\
\hline 3,3-Diethoxy-1-propyne & $10160-87-9$ \\
\hline trans- $\beta$-Farnesene & 18794-84-8 \\
\hline Octanal & $124-13-0$ \\
\hline Isobutyraldehyde & $78-84-2$ \\
\hline 3-Methylfuran & $930-27-8$ \\
\hline 1,1-Dimethylallyl alcohol & $115-18-4$ \\
\hline Butyl butyrate* & $109-21-7$ \\
\hline Acetaldehyde* & $75-07-0$ \\
\hline Menthone & $89-80-5$ \\
\hline Hexyl butyrate & $2639-63-6$ \\
\hline 2-Methylbutyraldehyde & $96-17-3$ \\
\hline (+)-Camphor & $464-49-3$ \\
\hline 1,4-Pentadiene & $591-93-5$ \\
\hline Terpinolene & $586-62-9$ \\
\hline Linalool oxide & 1365-19-1 \\
\hline 3-Octyl acetate & 4864-61-3 \\
\hline Geranyl acetate & $105-87-3$ \\
\hline
\end{tabular}




\begin{tabular}{|c|c|}
\hline Butyl acetate* & $123-86-4$ \\
\hline 3-Octanol & $589-98-0$ \\
\hline Decanal & $112-31-2$ \\
\hline 2-Methylfuran & $534-22-5$ \\
\hline Isobutyl methacrylate* & $97-86-9$ \\
\hline 2-Methyl-1-propene* & $115-11-7$ \\
\hline Borane-methyl sulfide complex & $13292-87-0$ \\
\hline Ethyl 1-methylcyclopropanecarboxylate & 71441-76-4 \\
\hline alpha-Santalene & $512-61-8$ \\
\hline 1-Octen-3-ol & 3391-86-4 \\
\hline 1,3,5,5-Tetramethyl-1,3-cyclohexadiene & 4724-89-4 \\
\hline (-)-trans-Pinocarveol & $547-61-5$ \\
\hline D,L-isobornyl acetate & $92618-89-8$ \\
\hline Lavandulyl acetate & $25905-14-0$ \\
\hline 2-Methylpropan-1-ol* & $78-83-1$ \\
\hline 1-Hexanol* & $111-27-3$ \\
\hline (S)-(-)-limonene oxide & 42477-94-1 \\
\hline cis-2-Pentene & $627-20-3$ \\
\hline 4-Methyl-1,3-pentadiene & $926-56-7$ \\
\hline 1-(1-Methylcyclopropyl)urea & 58102-14-0 \\
\hline 2-Ethylfuran & $3208-16-0$ \\
\hline Toluene* & $108-88-3$ \\
\hline cis-3-Hexene-1-ol & $928-96-1$ \\
\hline (E)-sabinene hydrate & 17699-16-0 \\
\hline cis-p-Menth-2,8-dienol & 22771-44-4 \\
\hline Butyl methyl ether & $628-28-4$ \\
\hline Butanone* & 78-93-3 \\
\hline (Z)-sabinene hydrate & $15537-55-0$ \\
\hline$( \pm)$-Citronellal & $106-23-0$ \\
\hline 4-Isopropyl-2-cyclohexenone & $500-02-7$ \\
\hline 2-Methyl-2-butene & 513-35-9 \\
\hline Isopropyl alcohol* & $67-63-0$ \\
\hline Methacrolein & 78-85-3 \\
\hline$( \pm)$-2-Methyl-1-butanol & $137-32-6$ \\
\hline Hexyl propionate & $2445-76-3$ \\
\hline Hexyl isobutyrate & 2349-07-7 \\
\hline alpha-Bergamotene & 17699-05-7 \\
\hline $\begin{array}{l}\text { 8-Isopropyl-1-methyl-5-methylene-1,6- } \\
\text { cyclodecadiene }\end{array}$ & 23986-74-5 \\
\hline Pentane* & $109-66-0$ \\
\hline Propanal* & $123-38-6$ \\
\hline Iso-propylcyclopropane & $3638-35-5$ \\
\hline trans-4-Tert-butylcyclohexan-1-ol & 21862-63-5 \\
\hline 2,7-Dimethyloxepine & $1487-99-6$ \\
\hline (-)-Caryophyllene oxide & $1139-30-6$ \\
\hline alpha-Santalol & $115-71-9$ \\
\hline Methyl acetate* & $79-20-9$ \\
\hline 2,3-Dimethylene-1,4-butanediol & $50521-50-1$ \\
\hline
\end{tabular}




\begin{tabular}{ll}
\hline Methyl isobutyl ketone* & $108-10-1$ \\
\hline Isoamyl alcohol & $123-51-3$ \\
\hline Methyl 2-ethylacrylate & $2177-67-5$ \\
\hline Undecane & $1120-21-4$ \\
\hline Butyl propionate & $590-01-2$ \\
\hline 3-Bromopentane & $1809-10-5$ \\
\hline Butyl isobutyrate & $97-87-0$ \\
\hline DL- $\alpha$-hydroxy- $\beta, \beta-d i m e t h y l-\gamma$-butyrolactone & $79-50-5$ \\
\hline Hexyl 2-methylbutanoate & $10032-15-2$ \\
\hline trans-Carveol & $1197-07-5$ \\
\hline 2-Methylpropyl ester* & $110-19-0$ \\
\hline 2-Butenoic acid, 2-methyl-, 2-methylpropyl ester & $66917-61-1$ \\
\hline *Classified as potentially hazardous &
\end{tabular}




\section{Mix essential oil}

\begin{tabular}{ll}
\hline Compounds & CAS\# \\
\hline Limonene* & $138-86-3$ \\
\hline alpha-Pinene & $80-56-8$ \\
\hline Linalool & $78-70-6$ \\
\hline Linalyl butyrate & $78-36-4$ \\
\hline Eucalyptol & $470-82-6$ \\
\hline beta-Pinene & $127-91-3$ \\
\hline Camphene & $79-92-5$ \\
\hline beta-Myrcene* & $123-35-3$ \\
\hline Benzyl acetate & $140-11-4$ \\
\hline Acetone* & $67-64-1$ \\
\hline beta-Phellandrene & $555-10-2$ \\
\hline Caryophyllene & $87-44-5$ \\
\hline Methyl benzoate & $93-58-3$ \\
\hline Borneol & $10385-78-1$ \\
\hline 3-Carene* & $13466-78-9$ \\
\hline alpha-Phellandrene & $99-83-2$ \\
\hline Isomenthone & $491-07-6$ \\
\hline Citronellyl formate & $105-85-1$ \\
\hline 3-Octanone & $106-68-3$ \\
\hline Hexyl acetate & $142-92-7$ \\
\hline beta-Citronellol & $106-22-9$ \\
\hline beta-trans-Ocimene & $3779-61-1$ \\
\hline Isopropyl alcohol* & $67-63-0$ \\
\hline Acetaldehyde* & $75-07-0$ \\
\hline Octanal & $124-13-0$ \\
\hline Geraniol & $106-24-1$ \\
\hline Neryl acetate & $141-12-8$ \\
\hline Isoborneol & $124-76-5$ \\
\hline Methanol* & $67-56-1$ \\
\hline 6-Methyl-5-hepten-2-one & $110-93-0$ \\
\hline Ethanol* & $64-17-5$ \\
\hline Geranyl acetate & $105-87-3$ \\
\hline Nonanal & $124-19-6$ \\
\hline Decanal & $112-31-2$ \\
\hline Methyl formate* & $107-31-3$ \\
\hline 1,4-Pentadiene & $591-93-5$ \\
\hline 2-Methylfuran & $534-22-5$ \\
\hline 3-Methylfuran & $930-27-8$ \\
\hline trans-Rose oxide & $876-18-6$ \\
\hline 8-Isopropyl-1-methyl-5-methylene-1,6- & $23986-74-5$ \\
\hline cyclodecadiene & \\
\hline 1,1-Dimethylallyl alcohol & $115-18-4$ \\
\hline Terpinolene & $586-62-9$ \\
\hline 3-Ethyl-2-methyl-1,3-heptadiene & $61142-35-6$ \\
\hline Hexyl butyrate & $2639-63-6$ \\
\hline & \\
\hline
\end{tabular}




\begin{tabular}{|c|c|}
\hline Linalool oxide & 1365-19-1 \\
\hline (Z)-linalool oxide (furanoid) & $5989-33-3$ \\
\hline alpha-Terpineol & $98-55-5$ \\
\hline 3-Ethyl-2-methyl-1,3-heptadiene & $61142-35-6$ \\
\hline 5-Isopropenyl-2-methylcyclohexanol & 18675-33-7 \\
\hline beta-Bourbonene & $5208-59-3$ \\
\hline Isobutyraldehyde & $78-84-2$ \\
\hline Methacrolein & $78-85-3$ \\
\hline 2,3-Dimethylpentane & $565-59-3$ \\
\hline 3-Methylhexane* & $589-34-4$ \\
\hline 1-(1-Methylcyclopropyl)urea & $58102-14-0$ \\
\hline 2-Ethylfuran & $3208-16-0$ \\
\hline Toluene* & $108-88-3$ \\
\hline Ethyl butyrate & $105-54-4$ \\
\hline Isopropylidenecyclohexane & $5749-72-4$ \\
\hline 2,6-Dimethylhepta-1,5-diene & $6709-39-3$ \\
\hline 2,2,6-Trimethyl-6-vinyltetrahydropyran & 7392-19-0 \\
\hline (E)-2,7-Dimethyl-3-octen-5-yne & 55956-33-7 \\
\hline$( \pm)$-Citronellal & $106-23-0$ \\
\hline Linalyl anthranilate & 7149-26-0 \\
\hline beta-Terpinyl acetate & 10198-23-9 \\
\hline alpha-Humulene & $6753-98-6$ \\
\hline delta-Cadinene & 483-76-1 \\
\hline (Z)-but-2-ene* & $590-18-1$ \\
\hline Pentane* & $109-66-0$ \\
\hline trans-1,2-Dimethylcyclopropane & $2402-06-4$ \\
\hline 2-Methyl-2-pentene & $625-27-4$ \\
\hline Butanone* & $78-93-3$ \\
\hline Ethyl acetate* & $141-78-6$ \\
\hline 2,3,4-Trimethylpentane* & $565-75-3$ \\
\hline 3-Ethyl-3-methylpentane* & $1067-08-9$ \\
\hline Isovaleraldehyde & $590-86-3$ \\
\hline Allyl ethyl carbonate & $1469-70-1$ \\
\hline Isoamyl alcohol & $123-51-3$ \\
\hline 4-Methyloctane & $2216-34-4$ \\
\hline 3-Methylcrotonaldehyde & $107-86-8$ \\
\hline 2,6-Dimethyl-1,3-heptadiene & 74779-65-0 \\
\hline 3-Methyl-1-pentanol & 42072-39-9 \\
\hline Butyl propionate & $590-01-2$ \\
\hline Butylbenzene & $104-51-8$ \\
\hline gamma-Terpinene & $99-85-4$ \\
\hline Propanal* & $123-38-6$ \\
\hline Methyl acetate* & $79-20-9$ \\
\hline 3-Methyl-1-cyclopentene & $1120-62-3$ \\
\hline 2,4-Hexadiene & $592-46-1$ \\
\hline 1,1-Dimethylcyclopentane & $1638-26-2$ \\
\hline 4,5-Dimethyloctane & $15869-96-2$ \\
\hline
\end{tabular}




\section{Tea tree essential oil}

\begin{tabular}{ll}
\hline Compounds & CAS\# \\
\hline (-)-Terpinen-4-ol & $20126-76-5$ \\
\hline gamma-Terpinene & $99-85-4$ \\
\hline 4-Carene & $29050-33-7$ \\
\hline m-Cymene & $535-77-3$ \\
\hline alpha-Pinene & $80-56-8$ \\
\hline Eucalyptol & $470-82-6$ \\
\hline Terpinolene & $586-62-9$ \\
\hline alpha-Phellandrene & $99-83-2$ \\
\hline Ethanol* & $64-17-5$ \\
\hline Limonene* & $138-86-3$ \\
\hline alpha-Terpineol & $98-55-5$ \\
\hline beta-Pinene & $127-91-3$ \\
\hline beta-Myrcene* & $123-35-3$ \\
\hline 3-Carene* & $13466-78-9$ \\
\hline Alloaromadendrene & $25246-27-9$ \\
\hline Isobutyraldehyde & $78-84-2$ \\
\hline alpha-Thujene & $2867-05-2$ \\
\hline (+)-Ledene & $21747-46-6$ \\
\hline delta-Cadinene & $483-76-1$ \\
\hline beta-Phellandrene & $555-10-2$ \\
\hline gamma-Muurolene & $30021-74-0$ \\
\hline Butane* & $106-97-8$ \\
\hline Acetone* & $67-64-1$ \\
\hline 2-Methylbutyraldehyde & $96-17-3$ \\
\hline alpha-Gurjunene & $489-40-7$ \\
\hline Caryophyllene & $87-44-5$ \\
\hline Methanol* & $67-56-1$ \\
\hline alpha-Copaene & $3856-25-5$ \\
\hline 2-Isopropenyltoluene & $7399-49-7$ \\
\hline Ethyl acetate* & $141-78-6$ \\
\hline Calamenene & $483-77-2$ \\
\hline Cycloheptane,4-methylene-1-methyl-2-(2-methyl- & $826337-63-7$ \\
\hline 1-propen-1-yl)-1-vinyl & \\
\hline 2-Methylpropan-1-ol* & $78-83-1$ \\
\hline (R)-(-)-2-butanol* & $14898-79-4$ \\
\hline (+)-Sativene & $3650-28-0$ \\
\hline 1,2,3,4,6,8a-Hexahydro-1-isopropyl-4,7-dimethyl- & $16728-99-7$ \\
\hline naphthalene & \\
\hline 2,5-Dimethyl-3-hexyne-2,5-diol & $142-30-3$ \\
\hline (-)-Isoledene & $95910-36-4$ \\
\hline beta-Cadinene & $523-47-7$ \\
\hline Camphene & $79-92-5$ \\
\hline (E)-sabinene hydrate & $17699-16-0$ \\
\hline 2,2-Dimethyl-4,5-di(1-propenyl)-1,3-dioxolane & $36334-88-0$ \\
\hline 1-Methyl-4-(1-methylethylidene)-2-(1- & $3242-08-8$ \\
\hline & \\
\hline
\end{tabular}




\begin{tabular}{|c|c|}
\hline \multicolumn{2}{|l|}{ methylvinyl)-1-vinylcyclohexane } \\
\hline Isovaleraldehyde & $590-86-3$ \\
\hline 1,4-Pentadiene & $591-93-5$ \\
\hline$( \pm)$-Citronellal & $106-23-0$ \\
\hline$(-)$-Borneol & $464-45-9$ \\
\hline cis-Carveol & 1197-06-4 \\
\hline Acetaldehyde* & $75-07-0$ \\
\hline$(-)$ - $\alpha$-Cubebene & $17699-14-8$ \\
\hline alpha-Gurjunene & $489-40-7$ \\
\hline$\gamma$-Gurjunene & $22567-17-5$ \\
\hline Butanone* & $78-93-3$ \\
\hline cis-3-Hexene-1-ol & $928-96-1$ \\
\hline Linalool & $78-70-6$ \\
\hline 3-Ethoxy-3,7-dimethylocta-1,6-diene & 72845-33-1 \\
\hline alpha-Humulene & $6753-98-6$ \\
\hline Rosifolio & 63891-61-2 \\
\hline Pentane* & $109-66-0$ \\
\hline 1,1-Dimethylallyl alcohol & $115-18-4$ \\
\hline (Z)-sabinene hydrate & $15537-55-0$ \\
\hline 1-Terpinenol & $586-82-3$ \\
\hline cis-p-Menth-1-en-3-ol & $16721-38-3$ \\
\hline Valencene & $4630-07-3$ \\
\hline 2-Butene* & $107-01-7$ \\
\hline 2-Methylfuran & $534-22-5$ \\
\hline 3-Methylfuran & $930-27-8$ \\
\hline ( \pm )-2-Methyl-1-butanol & $137-32-6$ \\
\hline 3-Methyl-2-pentanone & $565-61-7$ \\
\hline alpha-Fenchene & 471-84-1 \\
\hline (E)-linalool oxide (furanoid) & $34995-77-2$ \\
\hline Fenchyl alcohol & $1632-73-1$ \\
\hline 2-Ethylcyclohexanone & $4423-94-3$ \\
\hline$(-)-\beta$-Elemene & $515-13-9$ \\
\hline Spathulenol & $6750-60-3$ \\
\hline Tetracarbonylnickel* & $13463-39-3$ \\
\hline Glycolaldehyde dimer & 23147-58-2 \\
\hline Methyl acetate* & $79-20-9$ \\
\hline 2,3-Dimethyl-1-butene & $563-78-0$ \\
\hline 2-Butanol* & $78-92-2$ \\
\hline 2-Ethylfuran & $3208-16-0$ \\
\hline 2-Pentanone & $107-87-9$ \\
\hline 3-Pentanone* & $96-22-0$ \\
\hline Dimethyl disulfide & $624-92-0$ \\
\hline Isoamyl alcohol & $123-51-3$ \\
\hline 2,3,4-Trimethylhexane & $921-47-1$ \\
\hline Nonanol & $143-08-8$ \\
\hline 1-Hexanol* & $111-27-3$ \\
\hline$(-)$-Noe's reagent & 108031-79-4 \\
\hline 3-Ethyl-1,2-dimethylcyclohexa-1,4-diene & $125909-70-8$ \\
\hline
\end{tabular}




\begin{tabular}{ll}
\hline 3-Cyclohexene-1-carboxaldehyde & $100-50-5$ \\
\hline (S)-cis-Verbenol & $18881-04-4$ \\
\hline Camphene hydrate & $465-31-6$ \\
\hline 1-Methyl-4-isopropyl-1-cyclohexen-3-one & $89-81-6$ \\
\hline Isopentane* & $78-78-4$ \\
\hline trans-1,2-Dimethylcyclopropane & $2402-06-4$ \\
\hline 1-Methylcyclopentene & $693-89-0$ \\
\hline 1-Isopropoxy-2-propanol & $3944-36-3$ \\
\hline 1-Acetyl-2-methyl-1-cyclopentene & $3168-90-9$ \\
\hline 1,4-Dimethyl- $\delta$-3-tetrahydroacetophenone & $43219-68-7$ \\
\hline *Classified as potentially hazardous &
\end{tabular}




\section{Sweet orange essential oil}

\begin{tabular}{|c|c|}
\hline Compounds & CAS\# \\
\hline Limonene* & $138-86-3$ \\
\hline beta-Pinene & $127-91-3$ \\
\hline alpha-Pinene & $80-56-8$ \\
\hline beta-Phellandrene & $555-10-2$ \\
\hline 3-Carene* & $13466-78-9$ \\
\hline beta-trans-Ocimene & $3779-61-1$ \\
\hline Methanol* & $67-56-1$ \\
\hline Acetone* & 67-64-1 \\
\hline Octanal & $124-13-0$ \\
\hline Linalool & $78-70-6$ \\
\hline Ocimene & $13877-91-3$ \\
\hline Ethanol* & $64-17-5$ \\
\hline alpha-Thujene & $2867-05-2$ \\
\hline 1,1-Dimethylallyl alcohol & $115-18-4$ \\
\hline 1-Octanol* & $111-87-5$ \\
\hline (+)-Limonene oxide & $1195-92-2$ \\
\hline Methyl formate* & $107-31-3$ \\
\hline Decanal & $112-31-2$ \\
\hline alpha-Terpineol & $98-55-5$ \\
\hline gamma-Terpinene & $99-85-4$ \\
\hline (+)-(E)-limonene oxide & $6909-30-4$ \\
\hline Nonanal & $124-19-6$ \\
\hline 1-Hexanol* & $111-27-3$ \\
\hline alpha-Phellandrene & $99-83-2$ \\
\hline Camphene & $79-92-5$ \\
\hline Terpinolene & $586-62-9$ \\
\hline$( \pm)$-Citronellal & $106-23-0$ \\
\hline Acetaldehyde* & $75-07-0$ \\
\hline Butanone* & 78-93-3 \\
\hline Ethyl acetate* & $141-78-6$ \\
\hline 3-Methylhexane* & $589-34-4$ \\
\hline 4-Carene & 29050-33-7 \\
\hline Propanal* & $123-38-6$ \\
\hline Ethyl formate* & $109-94-4$ \\
\hline 2-Butanol* & $78-92-2$ \\
\hline Ethyl butyrate & $105-54-4$ \\
\hline Hexanal & $66-25-1$ \\
\hline 1-Heptanol* & $111-70-6$ \\
\hline 2-Ethenyl-1,1-dimethyl-3-methylenecyclohexane & 95452-08-7 \\
\hline 1-Octyl trifluoroacetate & $2561-21-9$ \\
\hline cis-p-Menth-2,8-dienol & 22771-44-4 \\
\hline Isoprene (stabilised)* & $78-79-5$ \\
\hline
\end{tabular}

*Classified as potentially hazardous 
Supplementary Table 2: All emitted VOCs from all essential oils $(n=14)$

\begin{tabular}{|c|c|c|}
\hline Compound & CAS \# & $\begin{array}{l}\text { Prevalence (\# } \\
\text { of Products) }\end{array}$ \\
\hline Acetaldehyde* & $75-07-0$ & 14 \\
\hline alpha-Phellandrene & $99-83-2$ & 14 \\
\hline alpha-Pinene & $80-56-8$ & 14 \\
\hline Camphene & $79-92-5$ & 14 \\
\hline Limonene* & $138-86-3$ & 14 \\
\hline Methanol* & $67-56-1$ & 14 \\
\hline Terpinolene & $586-62-9$ & 14 \\
\hline 3-Carene* & 13466-78-9 & 13 \\
\hline Acetone* & $67-64-1$ & 13 \\
\hline beta-Phellandrene & $555-10-2$ & 13 \\
\hline Ethanol* & $64-17-5$ & 13 \\
\hline gamma-Terpinene & $99-85-4$ & 13 \\
\hline alpha-Terpineol & $98-55-5$ & 12 \\
\hline beta-Pinene & $127-91-3$ & 12 \\
\hline Isovaleraldehyde & $590-86-3$ & 12 \\
\hline Linalool & $78-70-6$ & 12 \\
\hline beta-Myrcene* & $123-35-3$ & 11 \\
\hline Isobutyraldehyde & $78-84-2$ & 11 \\
\hline 1,1-Dimethylallyl alcohol & $115-18-4$ & 10 \\
\hline 2-Methylfuran & $534-22-5$ & 10 \\
\hline 3-Methylfuran & $930-27-8$ & 10 \\
\hline beta-trans-Ocimene & $3779-61-1$ & 10 \\
\hline Toluene* & 108-88-3 & 10 \\
\hline Caryophyllene & $87-44-5$ & 9 \\
\hline Eucalyptol & $470-82-6$ & 9 \\
\hline Pentane* & $109-66-0$ & 9 \\
\hline 2-Ethylfuran & $3208-16-0$ & 8 \\
\hline 2-Methylpropan-1-ol* & $78-83-1$ & 8 \\
\hline alpha-Thujene & 2867-05-2 & 8 \\
\hline Butanone* & 78-93-3 & 8 \\
\hline Ethyl acetate* & $141-78-6$ & 8 \\
\hline Isoamyl alcohol & $123-51-3$ & 8 \\
\hline m-Cymene & $535-77-3$ & 8 \\
\hline Neryl acetate & $141-12-8$ & 8 \\
\hline 2-Methyl-1-propene* & $115-11-7$ & 7 \\
\hline 2-Methylbutyraldehyde & $96-17-3$ & 7 \\
\hline
\end{tabular}


4-Carene

29050-33-7 7

Isopropyl alcohol*

67-63-0 7

Methyl acetate*

$79-20-9$

(Z)-sabinene hydrate

15537-55-0 6

1-Hexanol*

$111-27-3$

6

8-Isopropyl-1-methyl-5-methylene-1,6-

23986-74-5

6

cyclodecadiene

Geranyl acetate

105-87-3 6

Hexyl butyrate

2639-63-6

6

Lavandulyl acetate

25905-14-0

6

Methacrolein

78-85-3

6

Methyl isobutyl ketone*

108-10-1

6

Propanal*

123-38-6

6

$( \pm$ )-Citronellal

106-23-0

5

(E)-linalool oxide (furanoid)

$34995-77-2 \quad 5$

3-Octanone

106-68-3

4-Terpineol

562-74-3

5

alpha-Humulene

6753-98-6

Butyl butyrate*

109-21-7

Butyl propionate

590-01-2

cis-3-Hexene-1-ol

928-96-1

5

Hexyl acetate

142-92-7

Methyl formate*

107-31-3

(-)-Borneol

464-45-9

(-)-Terpinen-4-ol

20126-76-5

( \pm )-2-Methyl-1-butanol

137-32-6

(E)-sabinene hydrate

17699-16-0

1,4-Pentadiene

591-93-5

1-Octen-3-ol

3391-86-4

2442-10-6

1487-99-6

7399-49-7

500-02-7

17699-05-7

489-40-7

512-61-8

97-87-0

628-28-4

930-18-7

112-31-2

109-94-4

1632-73-1

5

Butyl methyl ether

cis-1,2-Dimethylcyclopropane

Decanal

Ethyl formate*

Fenchyl alcohol* 
Hexyl isobutyrate

2349-07-7 4

Hexyl methyl ether

4747-07-3 4

Hexyl propionate

2445-76-3 4

Iso-propylcyclopropane

3638-35-5 4

Linalool oxide

1365-19-1 4

Linalyl butyrate

78-36-4

Octanal

124-13-0

trans-1,2-Dimethylcyclopropane

2402-06-4 4

(-)-trans-Pinocarveol

547-61-5 3

(+)-Camphor

464-49-3

(Z)-beta-ocimene

3338-55-4

1-Pentene

109-67-1

2,3-Dimethyl-1-butene

563-78-0

2,4-Dimethylhexane*

589-43-5

2,4-Thujadiene

36262-09-6 3

2,5-Dimethylfuran

625-86-5

2-Methylpropyl ester*

110-19-0

2-Pentanone

107-87-9

3-Methylcrotonaldehyde

107-86-8

3-Methylhexane*

589-34-4

3-Octanol

589-98-0

6-Methyl-5-hepten-2-one

110-93-0

beta-Bourbonene

5208-59-3

Borneol

10385-78-1

Butane*

106-97-8

Butyl acetate*

123-86-4

Butylbenzene

104-51-8

Camphor

76-22-2

cis-4-Methyl-2-Pentene

691-38-3

delta-Cadinene

483-76-1

Ethyl butyrate

105-54-4

Hexanal

66-25-1

Hexane*

110-54-3

Isopentane*

78-78-4

Nonanal

124-19-6

trans-2-Methyl-1,3-pentadiene

926-54-5

trans- $\beta$-Farnesene

18794-84-8

(-)- $\alpha$-Cubebene

$17699-14-8$

$(+)$-Ledene

21747-46-6

$(+)$-Sativene

$3650-28-0$

(4E,6Z)-2,6-dimethylocta-2,4,6-triene

7216-56-0

(S)-cis-Verbenol

18881-04-4 
$\begin{array}{lll}\text { (Z)-but-2-ene* } & 590-18-1 & 2\end{array}$

(Z)-linalool oxide (furanoid) $\quad$ 5989-33-3 2

(Z)-rose oxide $\quad 16409-43-1 \quad 2$

1-(1-Methylcyclopropyl)urea $\quad 58102-14-0 \quad 2$

1-Acetyl-2-methyl-1-cyclopentene $\quad 3168-90-9 \quad 2$

1-Methyl-4-isopropyl-1-cyclohexen-3-one $\quad$ 89-81-6 2

1-Terpinenol 586-82-3 2

2-(1,1-Dimethyl-2-propenyl)-1,1- 81051-15-2 2

dimethylcyclopropane

2,2,6-Trimethyl-6-vinyltetrahydropyran $\quad 7392-19-0 \quad 2$

2,3,4-Trimethylhexane 921-47-1 2

2,6-Dimethylhepta-1,5-diene 6709-39-3 2

2-Butanol* 78-92-2 2

2-Butene* $107-01-7 \quad 2$

2-Methyl-1-butene $\quad 563-46-2 \quad 2$

2-Methyl-2-butene $\quad 513-35-9 \quad 2$

2-Methyl-2-pentene 625-27-4 2

2-Methylpentane* 107-83-5 2

3,4,4-Trimethyl-2-cyclopenten-1-one 30434-65-2 2

3-Ethyl-2-methyl-1,3-heptadiene 61142-35-6 2

3-Hexanone $\quad 589-38-8 \quad 2$

3-Methyl-1-cyclopentene $\quad 1120-62-3 \quad 2$

3-Octyl acetate 4864-61-3 2

3-Undecyne 60212-30-8 2

4,4,6,6-Tetramethylbicyclo[3.1.0]hex-2-ene 19487-09-3 2

4-Methyl-1,3-pentadiene $\quad 926-56-7 \quad 2$

Alloaromadendrene $\quad 25246-27-9 \quad 2$

alpha-Copaene 3856-25-5 2

alpha-Pinene oxide $\quad 1686-14-2 \quad 2$

alpha-Terpinene $\quad 99-86-5 \quad 2$

beta-Cadinene $\quad 523-47-7 \quad 2$

Bornyl acetate $\quad 76-49-3 \quad 2$

cis-Hexatriene 2612-46-6 2

cis-p-Menth-2,8-dienol 22771-44-4 2

Citronellyl acetate $\quad 150-84-5 \quad 2$

Citronellyl formate $\quad 105-85-1 \quad 2$

Cyclofenchene $\quad 488-97-1 \quad 2$

D,L-isobornyl acetate $\quad 92618-89-8 \quad 2$

D,L-Menthol 15356-70-4 2

Dimethyl sulfide $\quad 75-18-3 \quad 2$

gamma-Muurolene $\quad 30021-74-0 \quad 2$

Geraniol 106-24-1 2 
Hexyl 2-methylbutanoate

10032-15-2 2

Isoamyl acetate*

123-92-2 2

Isobutyl butyrate

539-90-2 2

Isoprene (stabilised)*

78-79-5 2

Isopropylidenecyclohexane

$5749-72-4 \quad 2$

Linalyl acetate

115-95-7

2

Menthol

1490-04-6 2

Menthone

89-80-5

2

Methyl (S)-2-methylbutanoate

53955-81-0 2

Ocimene

13877-91-3 2

Ocimenol

5986-38-9 2

o-Cymene

Pentylidenecyclopentane

527-84-4

2

53366-55-5 2

Perillen

$539-52-6$

2

Tetracarbonylnickel*

13463-39-3 2

trans-2-trans-4-Hexadiene

6108-61-8 2

(-)-Caryophyllene oxide

1139-30-6

1

(-)-Isoledene

95910-36-4

1

(-)-Noe's reagent

108031-79-4

1

$(-)$ - $\beta$-Elemene

515-13-9

1

$(+)$-(E)-limonene oxide

6909-30-4

(+)-Camphene

5794-03-6

1

(+)-Isomenthone

1196-31-2

(+)-Limonene oxide

1195-92-2

1

(+)-Longifolene

475-20-7

(1R,3R,4S)-2,2,3-

20536-41-8

1

1

trimethylbicyclo[2.2.1] heptane

22882-91-3 1

(2E)-1-ethoxy-3,7-dimethylocta-2,6-diene

(4E)-4-Hexenyl acetate

72237-36-6

(E)-2,7-Dimethyl-3-octen-5-yne

$55956-33-7$

1

141-27-5

(E)-citral*

19883-29-5

1

(E,E)-1,3,5-Undecatriene

14898-79-4

(R)-(-)-2-butanol*

6672-30-6

1

(R)-(+)-3-methylcyclopentanone

89-82-7

(R)-(+)-pulegone*

42477-94-1

70220-06-3

74630-62-9

4259-00-1

1638-26-2

16728-99-7

1,2,3,4,6,8a-Hexahydro-1-isopropyl-4,7-

dimethyl-naphthalene

(Z)-7-Methyl-5-undecene

1,1,2-Trimethylcyclopentane

1,1-Dimethylcyclopentane

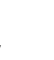

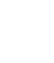

(1)

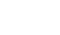

(1)

2

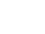

(1)


1,2,3-Trimethylcyclopentene

473-91-6 1

1,3,5,5-Tetramethyl-1,3-cyclohexadiene

4724-89-4 1

1,3-Cyclohexadiene

592-57-4 1

1,3-Dimethyl cyclohexene

2808-76-6 1

1,4-Dimethyl- $\delta$-3-tetrahydroacetophenone

43219-68-7 1

1-Acetyl-1-cyclohexene

932-66-1 1

1-Butyne

107-00-6 1

1-Cyclopropyl-1-pentanol

1-Heptanol*

4379-16-2 1

$111-70-6$

1-hydroxy-2-nitrocyclohexane-1-carboxylic

149695-77-2

1

acid

1-Isopropoxy-2-propanol

3944-36-3 1

1-Methoxy-2-propanol*

$107-98-2$

1-Methyl-3-(2-

methylcyclopropyl)cyclopropene

1-Methyl-3-isopropylcyclopentane

1-Methyl-3-propylcyclooctane

1-Methyl-4-(1-methylethylidene)-2-(1-

methylvinyl)-1-vinylcyclohexane

1-Methylcyclopentene

61142-26-5 1

1-Octanol*

1-Octyl trifluoroacetate

2-(2-Methyl-1-

propenyl)bicyclo[2.2.1]heptane

2,2,3-Trimethylhexane

2,2-Dimethyl-4,5-di(1-propenyl)-1,3-

dioxolane

2,2-Dimethylbutane*

2,3,4-Trimethylpentane*

2,3,5-Trimethyl-1,3-hexadiene

2,3-Dimethylcyclohexan-1-ol

2,3-Dimethylene-1,4-butanediol

2,3-Dimethylpentane

2,3-Pentadiene

53771-88-3 1

255885-37-1 1

3242-08-8 1

693-89-0 1

111-87-5 1

2561-21-9 1

61142-27-6 1

16747-25-4 1

36334-88-0 1

75-83-2 1

565-75-3 1

61142-34-5 1

1502-24-5 1

50521-50-1 1

565-59-3 1

591-96-8 1

2,4,6-Trimethyl-2,4,6-triphenyl-1,3,5,2,4,6- 546-45-2 1

trioxatrisilinane

2,4-Dimethyl-2,4-heptadiene $\quad$ 74421-05-9 1

2,4-Hexadiene

592-46-1 1

2,5-Diethyl tetrahydrofuran

2,5-Dimethyl-3-hexyne-2,5-diol

41239-48-9 1

$142-30-3 \quad 1$

2,5-Dimethylhex-5-en-3-yn-2-ol 2696-26-6 1

2,6-Dimethyl-1,3-heptadiene $\quad$ 74779-65-0 1

2,6-Dimethyl-3-heptene 2738-18-3 $\quad 1$

2[2(2-Methoxyethoxy)ethoxy]ethanol acetate $\quad 3610-27-3 \quad 1$ 
2-Bromocyclooctanone

39261-18-2 1

2-Butenoic acid, 2-methyl-, 2-methylpropyl

66917-61-1

ester

2-Ethenyl-1,1-dimethyl-3-

95452-08-7 1

methylenecyclohexane

2-Ethylcyclohexanone

2-Hexyne

4423-94-3 1

2-Isopropylidene-5-methylcyclohexanone

764-35-2

1

15932-80-6 1

2-Methoxy-1-methylethyl acetate*

108-65-6

2-Methyl-1-butanol

$34713-94-5$

1

2-Methyl-2-(4-methylpent-3-en-1-

yl)cyclopropylmethanol

2-Methylbutyl 2-methylbutyrate

98678-70-7

2-Methylbutyl acetate*

2445-78-5 1

624-41-9 1

2-Octynoic acid

3,3-Diethoxy-1-propyne

5663-96-7

10160-87-9 1

3,4-Dimethyl-2,4,6-octatriene

3,5-Heptadienal, 2-ethylidene-6-methyl-

57396-75-5 1

3,7-dimethyl-2,6-octadienyl isobutyrate

3,8-p-Menthadiene

3-Allylcyclohexene

99172-18-6 1

2345-26-8 1

586-67-4 1

15232-95-8 1

3-Bromopentane

3-Cyclohexene-1-carboxaldehyde

3-Ethoxy-3,7-dimethylocta-1,6-diene

1809-10-5 1

$100-50-5$

72845-33-1 1

125909-70-8 1

3-Ethyl-1,2-dimethylcyclohexa-1,4-diene

3-Ethyl-2,2-dimethyloxirane

1192-22-9 1

3-Ethyl-3-methylpentane*

1067-08-9

3-Methyl-1-pentanol

589-35-5

3-Methyl-1-pentanol

42072-39-9 1

3-Methyl-2-cyclopenten-1-one

2758-18-1

565-61-7

1

3-Methyl-2-pentanone

591-24-2

18729-48-1 1

3-Methylcyclopentanol

96-14-0

15232-76-5 1

3-Octyne

96-22-0

15869-95-1 1

4,4-Dimethyl octane

494-90-6

15869-96-2

72237-36-6

2216-34-4

18675-33-7

763-88-2

5-Methyl-1,4-hexadiene

4-Methyloctane

5-Isopropenyl-2-methylcyclohexanol

1

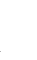

1

1

1

(1)

1

1

1

1

.

1

1


6-Bromocycloocta-1,4-diene

7-Methyl-1,6-octadiene

Acetaldehyde diethyl acetal*

Acetaldol

Acetylcyclohexane

Allyl ethyl carbonate

Allyl nonanoate

alpha-Fenchene

alpha-Santalol

alpha-Ylangene

Amyl isovalerate

Aristolene

Benzene*

Benzo(b)thiophene-1,1-dioxide

Benzyl acetate

beta-Bisabolene

beta-Citronellol

beta-Terpineol

beta-Terpinyl acetate

beta-Thujene

Bicyclo[4.1.0]hept-2-ene

Bicyclo[5.2.0]nonane, 2-methylene-4,8,8trimethyl-4-vinyl-

Borane-methyl sulfide complex

Bornylene

Butyl formate*

Butyraldehyde*

Calamenene

Camphene hydrate

Carane, 4,5-epoxy-, trans

cis-2-Pentene

cis-3,7-Dimethyl-2,6-octadien-1-ol

cis-3-Hexenyl butyrate

cis-3-Nonen-1-ol

cis-Carveol

cis-p-Menth-1-en-3-ol

Citronellyl butyrate

Citronellyl propionate

Cyclobutane

Cycloheptane,4-methylene-1-methyl-2-(2-

methyl-1-propen-1-yl)-1-vinyl

Cyclopentene,4-ethenyl-1,5,5-trimethyl
23359-89-9 1

42152-47-6 1

$105-57-7$

107-89-1 1

823-76-7

1469-70-1 1

7493-72-3 1

471-84-1 1

115-71-9 1

14912-44-8 1

25415-62-7 1

6831-16-9 1

71-43-2 1

825-44-5 1

140-11-4 1

495-61-4 1

106-22-9 1

138-87-4 1

10198-23-9 1

28634-89-1 1

2566-57-6 1

242794-76-9 1

13292-87-0 1

464-17-5 1

592-84-7 1

123-72-8 1

483-77-2 1

465-31-6 1

6909-20-2

627-20-3 1

106-25-2 1

16491-36-4 1

10340-23-5 1

1197-06-4 1

16721-38-3 1

141-16-2 1

141-14-0 1

287-23-0 1

826337-63-7 1

1727-69-1 


\begin{tabular}{|c|c|}
\hline Diethyl phthalate & 84-66-2 \\
\hline Dihydrocarveol & $619-01-2$ \\
\hline Dimethyl disulfide & $624-92-0$ \\
\hline Dimethyl phthalate* & $131-11-3$ \\
\hline Dl-Menthyl acetate & $16409-45-3$ \\
\hline DL- $\alpha$-hydroxy- $\beta, \beta$-dimethyl- $\gamma$-butyrolactone & $79-50-5$ \\
\hline Ethyl 1-methylcyclopropanecarboxylate & 71441-76-4 \\
\hline Ethyl 2-methylbutyrate & 7452-79-1 \\
\hline Ethyl 2-methylcyclopropanecarboxylate & 20913-25-1 \\
\hline Furan* & $110-00-9$ \\
\hline gamma-Terpineol & $586-81-2$ \\
\hline Geranyl butyrate & $106-29-6$ \\
\hline Geranyl propionate & $105-90-8$ \\
\hline Glycolaldehyde dimer & 23147-58-2 \\
\hline Hexyl formate & $629-33-4$ \\
\hline Iso-amyl-senecioate & $56922-73-7$ \\
\hline Isoborneol & $124-76-5$ \\
\hline Isobutyl methacrylate* & $97-86-9$ \\
\hline Isomenthone & $491-07-6$ \\
\hline Isopentyl formate* & $110-45-2$ \\
\hline Isopropyl butyrate & $638-11-9$ \\
\hline Isopropyl propionate* & $637-78-5$ \\
\hline Isopulegol & $7786-67-6$ \\
\hline Linalyl anthranilate & 7149-26-0 \\
\hline Linalyl isobutyrate & $78-35-3$ \\
\hline Menthone & $10458-14-7$ \\
\hline Methyl 2-ethylacrylate & $2177-67-5$ \\
\hline Methyl 3-methyl-2-butenoate & $924-50-5$ \\
\hline Methyl 3-methylvalerate & $2177-78-8$ \\
\hline Methyl 4-methylvalerate & $2412-80-8$ \\
\hline Methyl benzoate & $93-58-3$ \\
\hline Methyl butyrate & $623-42-7$ \\
\hline Methyl isopropyl ketone* & $563-80-4$ \\
\hline Methyl salicylate & $119-36-8$ \\
\hline Methyl valerate & $624-24-8$ \\
\hline Myrtanyl acetate & 29021-36-1 \\
\hline Nerol oxide & $1786-08-9$ \\
\hline Nonanol & $143-08-8$ \\
\hline Octyl acetate & $112-14-1$ \\
\hline $\mathrm{p}, \alpha$-Dimethylstyrene & $1195-32-0$ \\
\hline Pentanal & $110-62-3$ \\
\hline Phenethyl butyrate & $103-52-6$ \\
\hline
\end{tabular}




\begin{tabular}{lll} 
Phenyl butyrate & $4346-18-3$ & 1 \\
Propylene glycol & $57-55-6$ & 1 \\
Rosifolio & $63891-61-2$ & 1 \\
Sabinene & $3387-41-5$ & 1 \\
sec-Butyl acetate* & $105-46-4$ & 1 \\
Spathulenol & $6750-60-3$ & 1 \\
trans-2-Hexen-1-al & $6728-26-3$ & 1 \\
trans-3,4-Dimethyl-2-pentene & $4914-92-5$ & 1 \\
trans-4-Tert-butylcyclohexan-1-ol & $21862-63-5$ & 1 \\
trans-Carveol & $1197-07-5$ & 1 \\
trans-Rose oxide & $876-18-6$ & 1 \\
Undecane & $1120-21-4$ & 1 \\
Valencene & $4630-07-3$ & 1 \\
Vinyl methacrylate & $4245-37-8$ & 1 \\
Vinylcyclohexane & $695-12-5$ & 1 \\
$\beta$-Citronellol & $106-22-9$ & 1 \\
$\beta$-Selinene & $17066-67-0$ & 1 \\
$\gamma$-Gurjunene & $22567-17-5$ & 1 \\
\hline
\end{tabular}

*Compound classified as potentially hazardous 